Research Article

\title{
On Generalized Topological Indices of Silicon-Carbon
}

\author{
Xing-Long Wang, ${ }^{1}$ Jia-Bao Liu $\left(\mathbb{D},{ }^{2}\right.$ Akbar Jahanbani $\left(\mathbb{D},{ }^{3}\right.$ Muhammad Kamran Siddiqui $\left(\mathbb{D},{ }^{4}\right.$ \\ Nader Jafari Rad, ${ }^{5}$ and Roslan Hasni $\mathbb{C}^{6}$ \\ ${ }^{1}$ Department of General Education, Anhui Xinhua University, Hefei 230088, China \\ ${ }^{2}$ School of Mathematics and Physics, Anhui Jianzhu University, Hefei 230601, China \\ ${ }^{3}$ Department of Mathematics, Azarbaijan Shahid Madani University, Tabriz, Iran \\ ${ }^{4}$ Department of Mathematics, COMSATS University Islamabad, Lahore Campus, Islamabad, Pakistan \\ ${ }^{5}$ Department of Mathematics and Applications, Shahed University, Tehran, Iran \\ ${ }^{6}$ Faculty of Ocean Engineering Technology and Informatics, University Malaysia Terengganu, Kuala Nerus 21030 UMT, \\ Terengganu, Malaysia
}

Correspondence should be addressed to Muhammad Kamran Siddiqui; kamransiddiqui@cuilahore.edu.pk

Received 6 January 2020; Accepted 5 March 2020; Published 7 April 2020

Academic Editor: Mehdi Ghatee

Copyright (c) 2020 Xing-Long Wang et al. This is an open access article distributed under the Creative Commons Attribution License, which permits unrestricted use, distribution, and reproduction in any medium, provided the original work is properly cited.

\begin{abstract}
Let $G$ be a graph with $n$ vertices and $\Gamma(u)$ be the degree of its $u$-th vertex $(\Gamma(x)$ is the degree of $u)$. In this article, we compute the generalization of Zagreb index, the generalized Zagreb index, the first and second hyper $F$-indices, the sum connectivity $F$-index, and the product connectivity $F$-index graphs of $\mathrm{Si}_{2} \mathrm{C}_{3}-I[p, q], \mathrm{Si}_{2} \mathrm{C}_{3}-I I[p, q], \mathrm{Si}_{2} \mathrm{C}_{3}-I I I[p, q]$, and $\mathrm{SiC}_{3}-I I I[p, q]$.
\end{abstract}

\section{Introduction}

Mathematical chemistry is a branch of theoretical chemistry that discusses about the molecular structure by mathematical methods without necessarily referring to quantum mechanics. Molecular descriptors play a significant role in mathematical chemistry especially in QSPR/QSAR investigations [1]. A chemical structure can be represented by using graph theory, where vertices denote atoms and edges denote chemical bonds. Chemical graph theory is a branch of mathematical chemistry that it is a subject that connects mathematics, chemistry, and graph theory and solves problems arising in chemistry mathematically (for more details you can see [2-8]).

In chemical graph theory, a graph of molecule is a simple connected graph, in which atoms and chemical bonds are represented by vertices and edges, respectively. A graph is connected if there is a connection between any pair of vertices. Among them, special place is reserved for so-called topological descriptors or topological indices. Actually, topological indices are numeric quantities that tell us about the whole structure of graph. The topological indices are useful in the prediction of physicochemical properties and the bioactivity of the chemical compounds [9-11].

The topological indices of 2-dimensional silicon-carbons are computed in [12], in [13], Kwunet al. On the Multiplicative Degree-Based Topological Indices of Silicon-Carbon $\mathrm{Si}_{2} \mathrm{C}_{3}-I[p, q]$ and $S i_{2} \mathrm{C} 3-I I[p, q]$, in [14], Imran et al.. On Topological Properties of Symmetric Chemical Structures in [15], Idrees et al. Molecular Descriptors of Benzenoid System, in [16], Kulli. F-indices of Chemical Networks, in [6], in [17], Gao et al. the Redefined first, second and third Zagreb Indices of Titania Nanotubes $\mathrm{TiO}_{2}[m, n]$ and in [18], Kang et al. computed the topological indices of 2-dimensional silicon-carbon. For more details, see [19-23].

Bearing this in mind, it seems to be purposeful to compute the generalization of Zagreb index, the generalized Zagreb index, the first and second hyper $F$-indices, the sum connectivity $F$-index, and the product connectivity $F$-index of $S i_{2} C_{3}-I[p, q], S i_{2} C_{3}-I I[p, q], S i_{2} C_{3}-I I I[p, q]$, and $\mathrm{SiC}_{3}-\operatorname{III}[p, q]$. 
Throughout this paper, all graphs will be assumed simple that is without loops, multiple, or directed edges. Let $G=$ $(n, m)$ be a simple graph with vertex set $V(G)=\left\{v_{1}, v_{2}, v_{3}, \ldots, v_{n}\right\}$ and edge set $\mathbf{E}(G),|\mathbf{E}(G)|=m$. Also, let $d_{i}$ be the degree of vertex $v_{i}$ in graph $G$, for $i=1,2, \ldots, n$. The concept of valence in chemistry and the concept of degree in a graph are somehow closely related. For details on bases of graph theory, we refer to the book [24]. If two vertices $u$ and $v$ of the graph $G$ are adjacent, then the edge connecting them will be denoted by $u v$. The number of first neighbors of the vertex $u \in V(G)$ is its degree and will be denoted by $\Gamma(u)$.

In [25], the authors defined a new index, named generalization of Zagreb index:

$$
M_{\alpha, \beta}=M_{\alpha, \beta}(G)=\sum_{u v \in E(G)} \frac{(\Gamma(u) \times \Gamma(v))^{\alpha}}{(\Gamma(u)+\Gamma(v))^{\beta}},
$$

where $\alpha$ and $\beta$ are arbitrary real numbers. Few years later, the same index was proposed in [26] under the name second Gourava index, obtained as a special case of the generalized Zagreb index $M_{r, s}$ introduced in [27]:

$$
M_{r, s}=M_{r, s}(G)=\sum_{u v \in E(G)}\left[\left(\Gamma(u)^{r} \times \Gamma(v)^{s}\right)+\left(\Gamma(u)^{s} \times \Gamma(v)^{r}\right)\right] \text {. }
$$

In [28], Ghobadi et al. defined the hyper F-index or the first hyper F-index of a graph $G$ as

$$
H F_{1}(G)=\sum_{u v \in E(G)}\left(\Gamma(u)^{2}+\Gamma(v)^{2}\right)^{2}
$$

In [16], the second hyper F-index of a graph is defined as

$$
H F_{2}(G)=\sum_{u v \in E(G)}\left(\Gamma(u)^{2} \times \Gamma(v)^{2}\right)^{2} .
$$

In [16], the author introduced the sum connectivity F-index and the product connectivity F-index of a graph $G$, defined as

$$
\begin{aligned}
& S F(G)=\sum_{u v \in E(G)} \frac{1}{\sqrt{\Gamma(u)^{2}+\Gamma(v)^{2}}}, \\
& P F(G)=\sum_{u v \in E(G)} \frac{1}{\sqrt{\Gamma(u)^{2} \times \Gamma(v)^{2}}} .
\end{aligned}
$$

The concept of silicon carbide was introduced by an American scientist in 1891. But nowadays, we can produce silicon carbide artificially by silica and carbon. Till 1929, silicon carbide was known as the hardest material on Earth. Here, we will find out the generalization of Zagreb index, the generalized Zagreb index, the first and second hyper $F$-indices, the sum connectivity F-index, and the product connectivity F-index.

This paper is organized as follows. In Section 2, we compute the generalization of Zagreb index, the generalized Zagreb index, the first and second hyper $F$-indices, the sum connectivity F-index, and the product connectivity F-index graphs of $\mathrm{Si}_{2} \mathrm{C}_{3}-I[p, q]$. In Section 3, we compute the generalization of Zagreb index, the generalized Zagreb index, the first and second hyper F-indices, the sum connectivity $\mathrm{F}$-index, and the product connectivity F-index graphs of $\mathrm{Si}_{2} \mathrm{C}_{3}-I I[p, q]$. In Section 4 , we compute the generalization of Zagreb index, the generalized Zagreb index, the first and second hyper F-indices, the sum connectivity $\mathrm{F}$-index, and the product connectivity F-index graphs of $\mathrm{Si}_{2} \mathrm{C}_{3}-I I I[p, q]$. In Section 5 , we compute the generalization of Zagreb index, the generalized Zagreb index, the first and second hyper $F$-indices, the sum connectivity F-index, and the product connectivity F-index graphs of $\mathrm{SiC}_{3}-I I I[p, q]$.

For more details about these indices, see [12, 13, 29-39].

\section{Results for Silicon-Carbon $\mathrm{Si}_{2} \mathrm{C}_{3}-I[p, q]$}

In this section, we compute the generalization of Zagreb index, the generalized Zagreb index, the first and second hyper $F$-indices, the sum connectivity $\mathrm{F}$-index, and the product connectivity $\mathrm{F}$-index graphs of $\mathrm{Si}_{2} \mathrm{C}_{3}-I[p, q]$. In Figure 1, one unit of $S i_{2} C_{3}-I$ is shown. Molecular graph of $S_{i} 2 C_{3}-I$ is shown in Figure 2, in which $p$ denotes the number of cells attached in a single row and $q$ denotes the number of total rows where each row contains $p$ cells. In Figures 3 and 4, we demonstrate how cells are connected in one row (chain) and how one row is connected to another row. In Figures 1-4, carbon atoms are shown as brown, and silicon atoms $\mathrm{Si}$ are shown as blue.

Remark 1 (see [12]). The graph $\mathrm{Si}_{2} \mathrm{C}_{3}-I[p, q]$ contains $10 p q$ vertices and $15 p q-2 p-3 q$ edges.

We start by proving the carbon nanocones for the redefined Zagreb indices.

Theorem 1. Let $\mathrm{Si}_{2} \mathrm{C}_{3}-I[p, q]$ be the silicon carbide. Then,

$$
\begin{aligned}
M_{\alpha, \beta}\left(S i_{2} C_{3}-I[p, q]\right)= & 9^{\alpha} \times \frac{(15 p q-9 p-13 q+7)}{6^{\beta}}+6^{\alpha} \\
& \times \frac{(6 p+8 q-9)}{5^{\beta}}+(p+2 q) \\
& \times 4^{\alpha-\beta}+\frac{2^{\alpha}}{3^{\beta}}+\frac{3^{\alpha}}{4^{\beta}}, \\
M_{r, s}\left(S i_{2} C_{3}-I[p, q]\right)= & (6 p+8 q-9) \times\left(2^{r} \times 3^{s}+2^{s} \times 3^{r}\right) \\
& +(30 p q-18 p-26 q+14) \times 3^{s+r} \\
& +(2 p+4 q) \times 2^{s+r}+2^{r}+2^{s}+3^{r}+3^{s} .
\end{aligned}
$$

Proof. Consider the graph silicon carbide $\mathrm{Si}_{2} \mathrm{C}_{3}-I[p, q]$. By Remark 1, the graph $S_{2} C_{3}-I[p, q]$ contains $10 p q$ vertices and $15 p q-2 p-3 q$ edges. From the graph of $S_{2} C_{3}-I[p, q]$ silicon carbide, we can see that there are three partitions, $V_{\{1\}}=\left\{v \in V\left(S i_{2} C_{3}-I[p, q]\right) \mid \Gamma(v)=1\right\}, V_{\{2\}}=\left\{v \in V\left(S i_{2}\right.\right.$ 


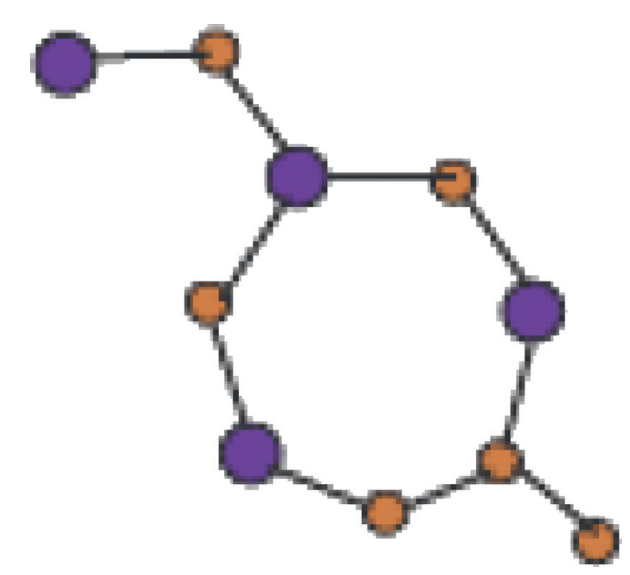

Figure 1: Unit cell of $S i_{2} C_{3}-I[p, q]$.

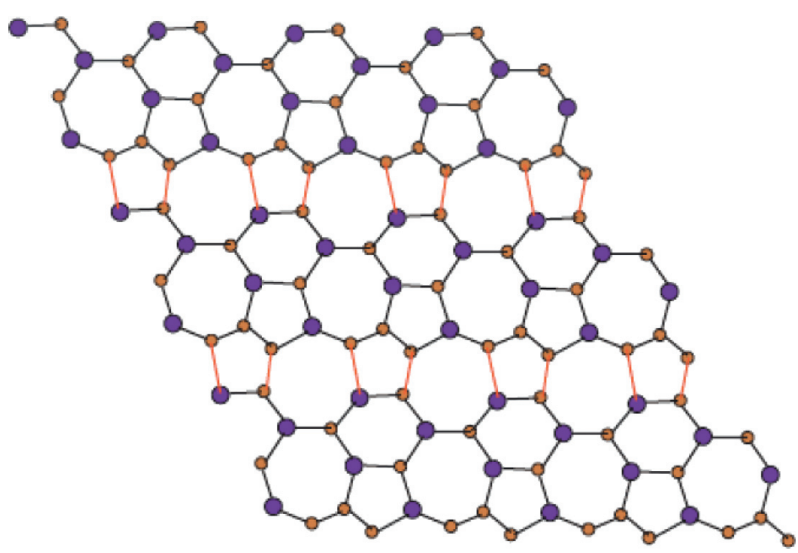

Figure 2: Sheet of $\mathrm{Si}_{2} \mathrm{C}_{3}-I[4,3]$.

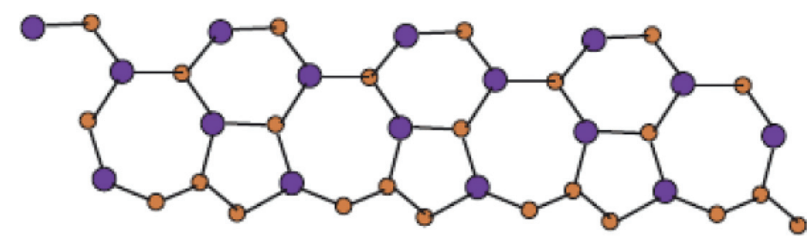

Figure 3: Sheet of $\mathrm{Si}_{2} \mathrm{C}_{3}-I[4,1]$.

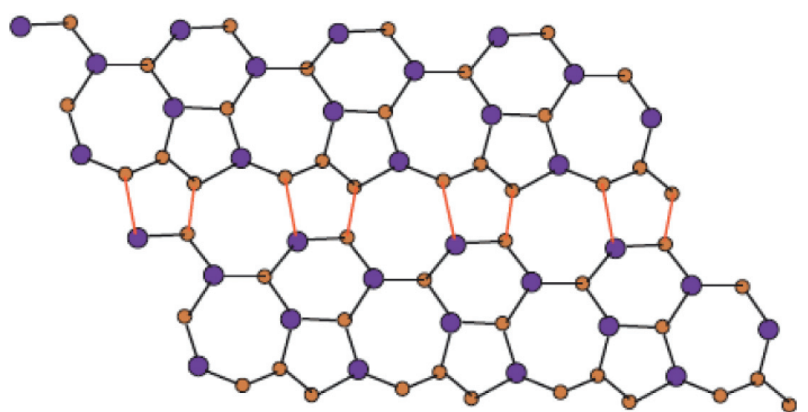

Figure 4: Sheet of $\mathrm{Si}_{2} \mathrm{C}_{3}-I[4,2]$.

$\left.\left.C_{3}-I[p, q]\right) \mid \Gamma(v)=2\right\}$, and $V_{\{3\}}=\left\{v \in V\left(S i_{2} C_{3}-I[p, q]\right)\right.$ $\mid \Gamma(v)=3\}$. The edge set of the $S i_{2} C_{3}-I[p, q]$ can be partitioned as follows:

$$
\begin{aligned}
& E_{1}=\left\{e=u v \in E\left(S i_{2} C_{3}-I[p, q]\right) \mid \Gamma(u)=1 \text { and } \Gamma(v)=2\right\}, \\
& E_{2}=\left\{e=u v \in E\left(S i_{2} C_{3}-I[p, q]\right) \mid \Gamma(u)=1 \text { and } \Gamma(v)=3\right\}, \\
& E_{3}=\left\{e=u v \in E\left(S i_{2} C_{3}-I[p, q]\right) \mid \Gamma(u)=2 \text { and } \Gamma(v)=2\right\}, \\
& E_{4}=\left\{e=u v \in E\left(S i_{2} C_{3}-I[p, q]\right) \mid \Gamma(u)=2 \text { and } \Gamma(v)=3\right\}, \\
& E_{5}=\left\{e=u v \in E\left(S i_{2} C_{3}-I[p, q]\right) \mid \Gamma(u)=3 \text { and } \Gamma(v)=3\right\} .
\end{aligned}
$$

From the molecular graph of $S i_{2} C_{3}-I[p, q]$, we can observe that $\left|E_{1}\right|=1,\left|E_{2}\right|=1,\left|E_{3}\right|=p+2 q,\left|E_{4}\right|=6 p-1+8$ $(q-1)$, and $\left|E_{3}\right|=15 p q-9 p-13 q+7$.

Thus, by definition generalization Zagreb index of $\mathrm{Si}_{2} \mathrm{C}_{3}-I[p, q]$, we have 


$$
\begin{aligned}
& M_{\alpha, \beta}\left(S i_{2} C_{3}-I[p, q]\right)=\sum_{u v \in E\left(S i_{2} C_{3}-I[p, q]\right)} \frac{(\Gamma(u) \times \Gamma(v))^{\alpha}}{(\Gamma(u)+\Gamma(v))^{\beta}}
\end{aligned}
$$

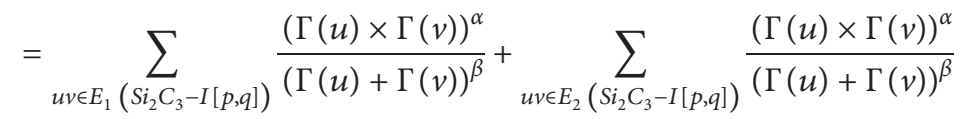

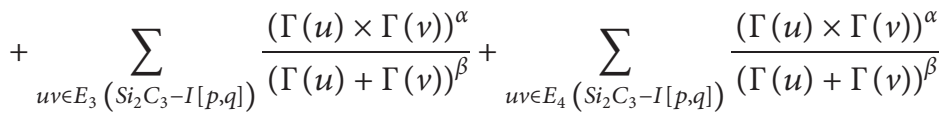

$$
\begin{aligned}
& +\sum_{u v \in E_{5}}\left(\sum_{\left.i_{2} C_{3}-I[p, q]\right)} \frac{(\Gamma(u) \times \Gamma(v))^{\alpha}}{(\Gamma(u)+\Gamma(v))^{\beta}}\right. \\
& =\frac{(1 \times 2)^{\alpha}}{(1+2)^{\beta}}+\frac{(1 \times 3)^{\alpha}}{(1+3)^{\beta}}+(p+2 q) \times \frac{(2 \times 2)^{\alpha}}{(2+2)^{\beta}} \\
& +(6 p-1+8(q-1)) \times \frac{(2 \times 3)^{\alpha}}{(2+3)^{\beta}}+(15 p q-9 p-13 q+7) \\
& \times \frac{(3 \times 3)^{\alpha}}{(3+3)^{\beta}}=9^{\alpha} \times \frac{(15 p q-9 p-13 q+7)}{6^{\beta}}+6^{\alpha} \times \frac{(6 p+8 q-9)}{5^{\beta}} \\
& +(p+2 q) \times 4^{\alpha-\beta}+\frac{2^{\alpha}}{3^{\beta}}+\frac{3^{\alpha}}{4^{\beta}} .
\end{aligned}
$$

which is the required (18) result.

By definition of the generalized Zagreb index of $\mathrm{Si}_{2} \mathrm{C}_{3}-I[p, q]$, we have

$$
\begin{aligned}
& M_{r, s}\left(S i_{2} C_{3}-I[p, q]\right)=\sum_{u v \in E\left(S i_{2} C_{3}-I[p, q]\right)}\left(\Gamma(u)^{r} \times \Gamma(v)^{s}+\Gamma(u)^{s} \times \Gamma(v)^{r}\right)
\end{aligned}
$$

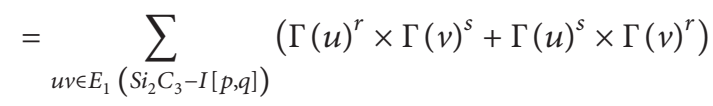

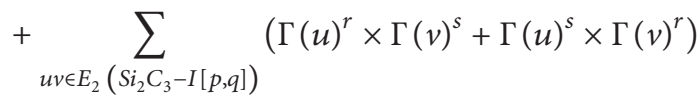

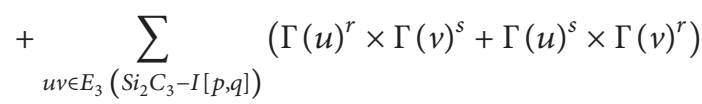

$$
\begin{aligned}
& +\sum_{u v \in E_{4}}\left(S i_{2} C_{3}-I[p, q]\right)\left(\Gamma(u)^{r} \times \Gamma(v)^{s}+\Gamma(u)^{s} \times \Gamma(v)^{r}\right)
\end{aligned}
$$

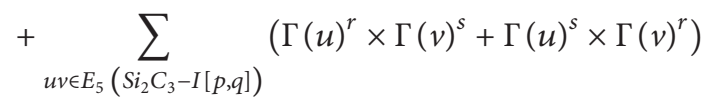

$$
\begin{aligned}
& =\left(1^{r} \times 2^{s}+1^{s} \times 2^{r}\right)+\left(1^{r} \times 3^{s}+1^{s} \times 3^{r}\right) \\
& +(p+2 q) \times\left(2^{r} \times 2^{s}+2^{s} \times 2^{r}\right)+(6 p-1+8(q-1)) \\
& \times\left(2^{r} \times 3^{s}+2^{s} \times 3^{r}\right)+(15 p q-9 p-13 q+7) \times\left(3^{r} \times 3^{s}+3^{s} \times 3^{r}\right) \\
& =(6 p+8 q-9) \times\left(2^{r} \times 3^{s}+2^{s} \times 3^{r}\right)+(30 p q-18 p-26 q+14) \times 3^{s+r} \\
& +(2 p+4 q) \times 2^{s+r}+2^{r}+2^{s}+3^{r}+3^{s} \text {. }
\end{aligned}
$$


which is the required (19) result.

Theorem 2. Let $\mathrm{Si}_{2} \mathrm{C}_{3}-I[p, q]$ be the silicon carbide. Then, $H F_{1}\left(S i_{2} C_{3}-I[p, q]\right)=4860 p q-1838 p-2732 q+872$,
Proof. Consider the graph silicon carbide $\mathrm{Si}_{2} \mathrm{C}_{3}-I[p, q]$. By Remark 1, the graph $\mathrm{Si}_{2} \mathrm{C}_{3}-I[p, q]$ contains $10 p q$ vertices and $15 p q-2 p-3 q$ edges. By definition of the first hyper F-index of $\mathrm{Si}_{2} \mathrm{C}_{3}-I[p, q]$, we have

$$
\mathrm{HF}_{2}\left(\mathrm{Si}_{2} \mathrm{C}_{3}-I[p, q]\right)=98415 p q-51017 p-74413 q+34360 .
$$

$$
\begin{aligned}
H F_{1}\left(S i_{2} C_{3}-I[p, q]\right)= & \sum_{u v \in E\left(S i_{2} C_{3}-I[p, q]\right)}\left(\Gamma(u)^{2}+\Gamma(v)^{2}\right)^{2} \\
= & \sum_{u v \in E_{1}\left(S i_{2} C_{3}-I[p, q]\right)}\left(\Gamma(u)^{2}+\Gamma(v)^{2}\right)^{2}+\sum_{u v \in E_{2}\left(S i_{2} C_{3}-I[p, q]\right)}\left(\Gamma(u)^{2}+\Gamma(v)^{2}\right)^{2} \\
& +\sum_{u v \in E_{3}} \sum_{\left(S i_{2} C_{3}-I[p, q]\right)}\left(\Gamma(u)^{2}+\Gamma(v)^{2}\right)^{2}+\sum_{u v \in E_{4}} \sum_{\left(S i_{2} C_{3}-I[p, q]\right)}\left(\Gamma(u)^{2}+\Gamma(v)^{2}\right)^{2} \\
& +\sum_{u v \in E_{5}}\left(S_{2} C_{3}-I[p, q]\right) \\
= & \left(1^{2}+2^{2}\right)^{2}+\left(1^{2}+3^{2}\right)^{2}+(p+2 q) \times\left(2^{2}+2^{2}\right)^{2}+\left(6 p-1+8(q)^{2}\right)^{2} \\
& \times\left(2^{2}+3^{2}\right)^{2}+(15 p q-9 p-13 q+7) \times\left(3^{2}+3^{2}\right)^{2} \\
= & 4860 p q-1838 p-2732 q+872 .
\end{aligned}
$$

which is the required (10) result.

By definition of the second hyper F-index of $\mathrm{Si}_{2} \mathrm{C}_{3}-I[p, q]$, we have

$$
\begin{aligned}
H F_{2}\left(S i_{2} C_{3}-I[p, q]\right)= & \sum_{u v \in E\left(S i_{2} C_{3}-I[p, q]\right)}\left(\Gamma(u)^{2}+\Gamma(v)^{2}\right)^{2} \\
= & \sum_{u v \in E_{1}\left(S i_{2} C_{3}-I[p, q]\right)}\left(\Gamma(u)^{2}+\Gamma(v)^{2}\right)^{2}+\sum_{u v \in E_{2}\left(S i_{2} C_{3}-I[p, q]\right)}\left(\Gamma(u)^{2}+\Gamma(v)^{2}\right)^{2} \\
& +\sum_{u v \in E_{3}\left(S i_{2} C_{3}-I[p, q]\right)}\left(\Gamma(u)^{2}+\Gamma(v)^{2}\right)^{2}+\sum_{u v \in E_{4}\left(S i_{2} C_{3}-I[p, q]\right)}\left(\Gamma(u)^{2}+\Gamma(v)^{2}\right)^{2} \\
& +\sum_{u v \in E_{5}}\left(S i_{2} C_{3}-I[p, q]\right) \\
= & \left.\left.\left(1^{2} \times 2^{2}\right)^{2}+\left(1^{2} \times 3^{2}\right)^{2}+(p+2 q) \times\left(2^{2} \times 2^{2}\right)^{2}+(6 p-1+8)^{2}\right)^{2}(q-1)\right) \\
& \times\left(2^{2} \times 3^{2}\right)^{2}+(15 p q-9 p-13 q+7) \times\left(3^{2} \times 3^{2}\right)^{2} \\
= & 98415 p q-51017 p-74413 q+34360 .
\end{aligned}
$$


which is the required (11) result.

Theorem 3. Let $\mathrm{Si}_{2} \mathrm{C}_{3}-I[p, q]$ be the silicon carbide. Then,

$$
\begin{aligned}
S F\left(S i_{2} C_{3}-I[p, q]\right)= & \sqrt{2} \times\left(\frac{5 p q}{2}-\frac{3 p}{2}-\frac{13 q}{6}+\frac{7}{6}\right)+\sqrt{2} \\
& \times\left(\frac{p q}{4}+\frac{q}{2}\right)+\sqrt{13} \times\left(\frac{6 p+8 q-9}{13}+\frac{q}{2}\right) \\
& +\frac{\sqrt{5}}{5}+\frac{\sqrt{10}}{10},
\end{aligned}
$$$$
P F\left(S i_{2} C_{3}-I[p, q]\right)=\frac{9 k n^{2}}{4}-\frac{27 k n}{20}+\frac{k}{10} .
$$

Proof. Consider the graph silicon carbide $\mathrm{Si}_{2} \mathrm{C}_{3}-I[p, q]$. By Remark 1 , the graph $S_{2} C_{3}-I[p, q]$ contains $10 p q$ vertices and $15 p q-2 p-3 q$ edges. By definition of the sum connectivity F-index of $S_{2} C_{3}-I[p, q]$, we have

$$
\begin{aligned}
& S F\left(S i_{2} C_{3}-I[p, q]\right)=\sum_{u v \in E\left(S i_{2} C_{3}-I[p, q]\right)} \frac{1}{\sqrt{\Gamma(u)^{2}+\Gamma(v)^{2}}} \\
& =\sum_{u v \in E_{1}} \frac{1}{\left(S i_{2} C_{3}-I[p, q]\right)}+\sum_{\sqrt{\Gamma(u)^{2}+\Gamma(v)^{2}}}+\frac{1}{\frac{1}{\sqrt{\Gamma(u)^{2}+\Gamma(v)^{2}}}} \\
& +\sum_{u v \in E_{3}\left(S i_{2} C_{3}-I[p, q]\right)} \frac{1}{\sqrt{\Gamma(u)^{2}+\Gamma(v)^{2}}}+\sum_{u v \in E_{4}} \sum_{\left(S i_{2} C_{3}-I[p, q]\right)} \frac{1}{\sqrt{\Gamma(u)^{2}+\Gamma(v)^{2}}} \\
& +\sum_{u v \in E_{5}} \frac{1}{\left(S i_{2} C_{3}-I[p, q]\right)} \frac{1}{\sqrt{\Gamma(u)^{2}+\Gamma(v)^{2}}} \\
& =\frac{1}{\sqrt{1^{2}+2^{2}}}+\frac{1}{\sqrt{1^{2}+3^{2}}}+(p+2 q) \times\left(\frac{1}{\sqrt{2^{2}+2^{2}}}\right)+(6 p-1+8(q-1)) \\
& \times\left(\frac{1}{\sqrt{2^{2}+3^{2}}}\right)+(15 p q-9 p-13 q+7) \times\left(\frac{1}{\sqrt{3^{2}+3^{2}}}\right) \\
& =\sqrt{2} \times\left(\frac{5 p q}{2}-\frac{3 p}{2}-\frac{13 q}{6}+\frac{7}{6}\right)+\sqrt{2} \times\left(\frac{p q}{4}+\frac{q}{2}\right) \\
& +\sqrt{13} \times\left(\frac{6 p+8 q-9}{13}+\frac{q}{2}\right)+\frac{\sqrt{5}}{5}+\frac{\sqrt{10}}{10} .
\end{aligned}
$$

which is the required (14) result.

By definition of product connectivity F-index of $\mathrm{Si}_{2} \mathrm{C}_{3}-I[p, q]$, we have 


$$
\begin{aligned}
& P F\left(S i_{2} C_{3}-I[p, q]\right)=\sum_{u v \in E\left(S i_{2} C_{3}-I[p, q]\right)} \frac{1}{\sqrt{\Gamma(u)^{2}+\Gamma(v)^{2}}} \\
& =\sum_{u v \in E_{1}} \frac{1}{\left(S i_{2} C_{3}-I[p, q]\right)} \frac{1}{\sqrt{\Gamma(u)^{2}+\Gamma(v)^{2}}}+\sum_{u v \in E_{2}} \frac{1\left(S i_{2} C_{3}-I[p, q]\right)}{\frac{\sqrt{\Gamma(u)^{2}+\Gamma(v)^{2}}}{}} \\
& +\sum_{u v \in E_{3}} \frac{1}{\left(S i_{2} C_{3}-I[p, q]\right)} \frac{1}{\sqrt{\Gamma(u)^{2}+\Gamma(v)^{2}}}+\sum_{u v \in E_{4}}\left(S i_{2} C_{3}-I[p, q]\right) \frac{1}{\sqrt{\Gamma(u)^{2}+\Gamma(v)^{2}}} \\
& +\sum_{u v \in E_{5}}\left(S i_{2} C_{3}-I[p, q]\right) \frac{1}{\sqrt{\Gamma(u)^{2}+\Gamma(v)^{2}}} \\
& =\frac{1}{\sqrt{1^{2} \times 2^{2}}}+\frac{1}{\sqrt{1^{2} \times 3^{2}}}+(p+2 q) \times\left(\frac{1}{\sqrt{2^{2} \times 2^{2}}}\right)+(6 p-1+8(q-1)) \\
& \times\left(\frac{1}{\sqrt{2^{2} \times 3^{2}}}\right)+(15 p q-9 p-13 q+7) \times\left(\frac{1}{\sqrt{3^{2} \times 3^{2}}}\right) \\
& =\frac{5 p q}{3}+\frac{p}{4}+\frac{7 q}{18}+\frac{1}{9} .
\end{aligned}
$$

which is the required (15) result.

\section{Results for Silicon-Carbon $\mathrm{Si}_{2} \mathrm{C}_{3}-I I[p, q]$}

In this section, we compute the generalization of Zagreb index, the generalized Zagreb index, the first and second hyper $F$-indices, the sum connectivity $\mathrm{F}$-index, and the product connectivity F-index graphs of $\mathrm{Si}_{2} \mathrm{C}_{3}-I I[p, q]$.In Figure 5, one unit of $S i_{2} C_{3}-I I$ is given. By connecting $p$ cells in a row and then connecting $q$ rows where each row contains $p$ cells, we get molecular graph of $\mathrm{Si}_{2} \mathrm{C}_{3}-I I$. The molecular graph of $\mathrm{Si}_{2} \mathrm{C}_{3}-I I$ is shown in Figure 6 for $p=3$ and $q=4$. Figures 7 and 8 demonstrate how cells are connected in a row (chain) and how a row is connected to another row. We will use $\mathrm{Si}_{2} \mathrm{C}_{3}-I I[p, q]$ to represent this molecular graph.

Remark 2 (see [12]). The graph $\mathrm{Si}_{2} \mathrm{C}_{3}-I I[p, q]$ contains $10 p q$ vertices and $15 p q-3 p-3 q$ edges.

We start by proving the silicon carbide for the generalization of Zagreb index.

Theorem 4. Let $\mathrm{Si}_{2} \mathrm{C}_{3}-I I[p, q]$ be the silicon carbide. Then,

$$
\begin{aligned}
M_{\alpha, \beta}\left(S i_{2} C_{3}-I I[p, q]\right)= & 9^{\alpha} \times \frac{(15 p q-9 p-13 q+7)}{6^{\beta}} \\
& +6^{\alpha} \times \frac{(6 p+8 q-9)}{5^{\beta}}+(p+2 q) \\
& \times 4^{\alpha-\beta}+\frac{2^{\alpha}}{3^{\beta}}+\frac{3^{\alpha}}{4^{\beta}}
\end{aligned}
$$

$$
\begin{aligned}
M_{r, s}\left(S i_{2} C_{3}-I I[p, q]\right)= & (6 p+8 q-9) \times\left(2^{r} \times 3^{s}+2^{s} \times 3^{r}\right) \\
& +(30 p q-18 p-26 q+14) \times 3^{s+r} \\
& +(2 p+4 q) \times 2^{s+r}+2^{r}+2^{s}+3^{r}+3^{s}
\end{aligned}
$$

Proof. Consider the graph silicon carbide $\mathrm{Si}_{2} \mathrm{C}_{3}-I I[p, q]$. By Remark 2, the graph $S i_{2} C_{3}-I I[p, q]$ contains $10 p q$ vertices and $15 p q-2 p-3 q$ edges. From the graph of $\mathrm{Si}_{2} \mathrm{C}_{3}-I I[p, q]$ silicon carbide, we can see that there are three partitions, $V_{\{1\}}=\left\{v \in V\left(S i_{2} C_{3}-I I[p, q]\right) \mid \Gamma(v)=1\right\}$, $V_{\{2\}}=\left\{v \in V\left(S i_{2} C_{3}-I I[p, q]\right) \mid \Gamma(v)=2\right\}$, and $V_{\{3\}}=\{v \in$ $\left.V\left(S i_{2} C_{3}-I I[p, q]\right) \mid \Gamma(v)=3\right\}$. The edge set of the $S i_{2} C_{3}-$ $I I[p, q]$ can be partitioned as follows: 


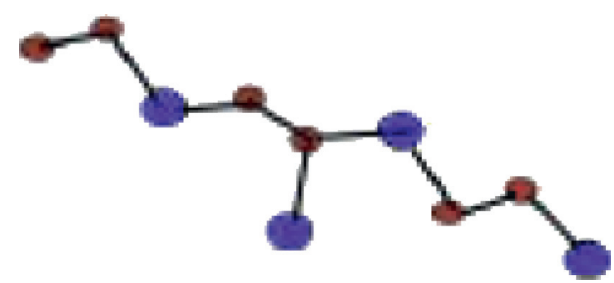

Figure 5: One unit of $\mathrm{Si}_{2} \mathrm{C}_{3}-I I[p, q]$.

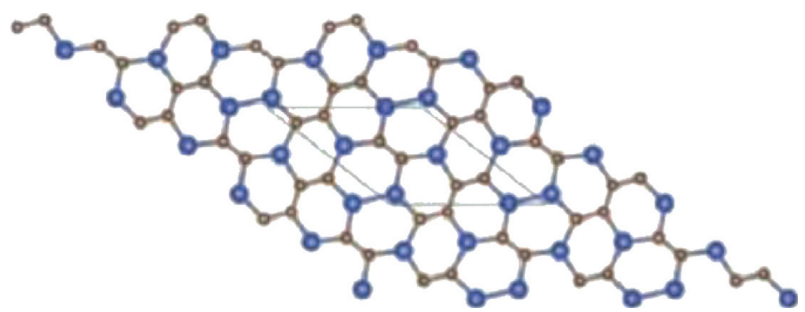

Figure 6: Sheet of $\mathrm{Si}_{2} \mathrm{C}_{3}-I I[3,3]$.

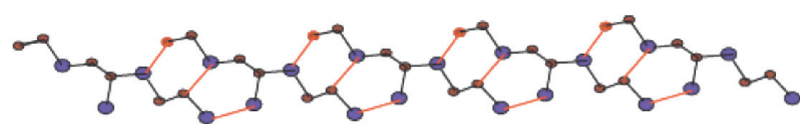

Figure 7: Sheet of $\mathrm{Si}_{2} \mathrm{C}_{3}-I I[5,1]$.

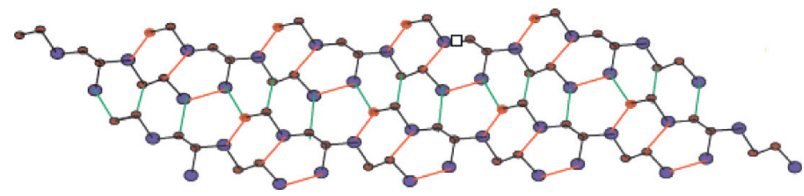

Figure 8: Sheet of $\mathrm{Si}_{2} \mathrm{C}_{3}-I I[5,2]$.

$$
\begin{aligned}
& E_{1}=\left\{e=u v \in E\left(S i_{2} C_{3}-I I[p, q]\right) \mid \Gamma(u)=1 \text { and } \Gamma(v)=2\right\}, \\
& E_{2}=\left\{e=u v \in E\left(S i_{2} C_{3}-I I[p, q]\right) \mid \Gamma(u)=1 \text { and } \Gamma(v)=3\right\}, \\
& E_{3}=\left\{e=u v \in E\left(S i_{2} C_{3}-I I[p, q]\right) \mid \Gamma(u)=2 \text { and } \Gamma(v)=2\right\}, \\
& E_{4}=\left\{e=u v \in E\left(S i_{2} C_{3}-I I[p, q]\right) \mid \Gamma(u)=2 \text { and } \Gamma(v)=3\right\}, \\
& E_{5}=\left\{e=u v \in E\left(S i_{2} C_{3}-I I[p, q]\right) \mid \Gamma(u)=3 \text { and } \Gamma(v)=3\right\} .
\end{aligned}
$$

From the molecular graph of $\mathrm{Si}_{2} \mathrm{C}_{3}-I I[p, q]$, we can observe that $\left|E_{1}\right|=2,\left|E_{2}\right|=1,\left|E_{3}\right|=2 p+2 q,\left|E_{4}\right|=8 p+8 q$ -14 , and $\left|E_{3}\right|=15 p q-13 p-13 q+11$. Thus, by definition generalization of $\mathrm{Si}_{2} \mathrm{C}_{3}-I I[p, q]$, we

$$
\begin{aligned}
M_{\alpha, \beta}\left(S i_{2} C_{3}-I[p, q]\right)= & \sum_{u v \in E\left(S i_{2} C_{3}-I[p, q]\right)} \frac{(\Gamma(u) \times \Gamma(v))^{\alpha}}{(\Gamma(u)+\Gamma(v))^{\beta}} \\
= & \sum_{u v \in E_{1}\left(S i_{2} C_{3}-I[p, q]\right)} \frac{(\Gamma(u) \times \Gamma(v))^{\alpha}}{(\Gamma(u)+\Gamma(v))^{\beta}}+\sum_{u v \in E_{2}\left(S i_{2} C_{3}-I[p, q]\right)} \frac{(\Gamma(u) \times \Gamma(v))^{\alpha}}{(\Gamma(u)+\Gamma(v))^{\beta}} \\
& +\sum_{u v \in E_{3}\left(S i_{2} C_{3}-I[p, q]\right)} \frac{(\Gamma(u) \times \Gamma(v))^{\alpha}}{(\Gamma(u)+\Gamma(v))^{\beta}}+\sum_{u v \in E_{4}\left(S i_{2} C_{3}-I[p, q]\right)} \frac{(\Gamma(u) \times \Gamma(v))^{\alpha}}{(\Gamma(u)+\Gamma(v))^{\beta}} \\
& +\sum_{u v \in E_{5}\left(S i_{2} C_{3}-I[p, q]\right)} \frac{(\Gamma(u) \times \Gamma(v))^{\alpha}}{(\Gamma(u)+\Gamma(v))^{\beta}} \\
= & 2 \times \frac{(1 \times 2)^{\alpha}}{(1+2)^{\beta}}+\frac{(1 \times 3)^{\alpha}}{(1+3)^{\beta}}+(2 p+2 q) \times \frac{(2 \times 2)^{\alpha}}{(2+2)^{\beta}} \\
& +(8 p+8 q-14) \times \frac{(2 \times 3)^{\alpha}}{(2+3)^{\beta}+(15 p q-13 p-13 q+11)} \\
& \times \frac{(3 \times 3)^{\alpha}}{(3+3)^{\beta}}=9^{\alpha} \times \frac{(15 p q-13 p-13 q+11)}{6^{\beta}}+6^{\alpha} \times \frac{(8 p+8 q-14)}{5^{\beta}} \\
& +(2 p+2 q) \times 4^{\alpha-\beta}+2 \times \frac{2^{\alpha}}{3^{\beta}}+\frac{3^{\alpha}}{4^{\beta}} .
\end{aligned}
$$

which is the required (18) result. 
By definition of the generalized Zagreb index of $\mathrm{Si}_{2} \mathrm{C}_{3}-I I[p, q]$, we have

$$
\begin{aligned}
M_{r, s}\left(S i_{2} C_{3}-I[p, q]\right)= & \sum_{u v \in E\left(S i_{2} C_{3}-I[p, q]\right)}\left(\Gamma(u)^{r} \times \Gamma(v)^{s}+\Gamma(u)^{s} \times \Gamma(v)^{r}\right) \\
= & \sum_{u v \in E_{1}\left(S i_{2} C_{3}-I[p, q]\right)}\left(\Gamma(u)^{r} \times \Gamma(v)^{s}+\Gamma(u)^{s} \times \Gamma(v)^{r}\right) \\
& +\sum_{u v \in E_{2}\left(S i_{2} C_{3}-I[p, q]\right)}\left(\Gamma(u)^{r} \times \Gamma(v)^{s}+\Gamma(u)^{s} \times \Gamma(v)^{r}\right) \\
& +\sum_{u v \in E_{3}\left(S i_{2} C_{3}-I[p, q]\right)}\left(\Gamma(u)^{r} \times \Gamma(v)^{s}+\Gamma(u)^{s} \times \Gamma(v)^{r}\right) \\
& +\sum_{u v \in E_{4}\left(S i_{2} C_{3}-I[p, q]\right)}\left(\Gamma(u)^{r} \times \Gamma(v)^{s}+\Gamma(u)^{s} \times \Gamma(v)^{r}\right) \\
& +\sum_{u v \in E_{5}\left(s i_{2} C_{3}-I[p, q]\right)}\left(\Gamma(u)^{r} \times \Gamma(v)^{s}+\Gamma(u)^{s} \times \Gamma(v)^{r}\right) \\
= & 2 \times\left(1^{r} \times 2^{s}+1^{s} \times 2^{r}\right)+\left(1^{r} \times 3^{s}+1^{s} \times 3^{r}\right) \\
& +(2 p+2 q) \times\left(2^{r} \times 2^{s}+2^{s} \times 2^{r}\right)+(8 p+8 q-14) \\
& \times\left(2^{r} \times 3^{s}+2^{s} \times 3^{r}\right)+(15 p q-13 p-13 q+11) \times\left(3^{r} \times 3^{s}+3^{s} \times 3^{r}\right) \\
= & (8 p-6) \times\left(2^{r} \times 3^{s}+2^{s} \times 3^{r}\right)+(30 p q-26 p-26 q+22) \times 3^{s+r} \\
& +(4 p+4 q) \times 2^{s+r}+2 \times 2^{r}+2 \times 2^{s}+3^{r}+3^{s} .
\end{aligned}
$$

which is the required (19) result.

Theorem 5. Let $\mathrm{Si}_{2} \mathrm{C}_{3}-I I[p, q]$ be the silicon carbide. Then, $H F_{1}\left(S i_{2} C_{3}-I I[p, q]\right)=4860 p q-2732 p-2732 q+1348$,
Proof. Consider the graph silicon carbide $\mathrm{Si}_{2} \mathrm{C}_{3}-I I[p, q]$. By Remark 2, the graph $\mathrm{Si}_{2} \mathrm{C}_{3}-I I[p, q]$ contains $10 p q$ vertices and $15 p q-2 p-3 q$ edges. By definition of the first hyper F-index of $\mathrm{Si}_{2} \mathrm{C}_{3}-I I[p, q]$, we have

$H_{2}\left(S i_{2} C_{3}-I I[p, q]\right)=98415 p q-74413 p-74413 q+54140$.

$$
\begin{aligned}
H F_{1}\left(S i_{2} C_{3}-I I[p, q]\right)= & \sum_{u v \in E\left(S i_{2} C_{3}-I I[p, q]\right)}\left(\Gamma(u)^{2}+\Gamma(v)^{2}\right)^{2} \\
= & \sum_{u v \in E_{1}\left(S i_{2} C_{3}-I I[p, q]\right)}\left(\Gamma(u)^{2}+\Gamma(v)^{2}\right)^{2}+\sum_{u v \in E_{2}\left(S i_{2} C_{3}-I I[p, q]\right)}\left(\Gamma(u)^{2}+\Gamma(v)^{2}\right)^{2} \\
& +\sum_{u v \in E_{3}\left(S i_{2} C_{3}-I I[p, q]\right)}\left(\Gamma(u)^{2}+\Gamma(v)^{2}\right)^{2}+\sum_{u v \in E_{4}\left(S_{2} C_{3}-I I[p, q]\right)}\left(\Gamma(u)^{2}+\Gamma(v)^{2}\right)^{2} \\
& +\sum_{u v \in E_{5}}\left(\sum_{\left.i_{2} C_{3}-I I[p, q]\right)}\left(\Gamma(u)^{2}+\Gamma(v)^{2}\right)^{2}\right. \\
= & 2 \times\left(1^{2}+2^{2}\right)^{2}+\left(1^{2}+3^{2}\right)^{2}+(2 p+2 q) \times\left(2^{2}+2^{2}\right)^{2}+(8 p+8 q-14) \\
& \times\left(2^{2}+3^{2}\right)^{2}+(15 p q-13 p-13 q+11) \times\left(3^{2}+3^{2}\right)^{2} \\
= & 4860 p q-2732 p-2732 q+1348 .
\end{aligned}
$$


which is the required (23) result.

By definition of the second hyper F-index of $\mathrm{Si}_{2} \mathrm{C}_{3}-I I[p, q]$, we have

$$
\begin{aligned}
H F_{2}\left(S i_{2} C_{3}-I I[p, q]\right)= & \sum_{u v \in E\left(S i_{2} C_{3}-I I[p, q]\right)}\left(\Gamma(u)^{2} \times \Gamma(v)^{2}\right)^{2} \\
= & \sum_{u v \in E_{1}\left(S i_{2} C_{3}-I I[p, q]\right)}\left(\Gamma(u)^{2} \times \Gamma(v)^{2}\right)^{2}+\sum_{u v \in E_{2}\left(S i_{2} C_{3}-I I[p, q]\right)}\left(\Gamma(u)^{2} \times \Gamma(v)^{2}\right)^{2} \\
& +\sum_{u v \in E_{3}\left(S i_{2} C_{3}-I I[p, q]\right)}\left(\Gamma(u)^{2} \times \Gamma(v)^{2}\right)^{2}+\sum_{u v \in E_{4}\left(S i_{2} C_{3}-I I[p, q]\right)}\left(\Gamma(u)^{2} \times \Gamma(v)^{2}\right)^{2} \\
& +\sum_{u v \in E_{5}\left(S i_{2} C_{3}-I I[p, q]\right)}\left(\Gamma(u)^{2} \times \Gamma(v)^{2}\right)^{2} \\
= & 2 \times\left(1^{2} \times 2^{2}\right)^{2}+\left(1^{2} \times 3^{2}\right)^{2}+(2 p+2 q) \times\left(2^{2} \times 2^{2}\right)^{2}+(8 p+8 q-14) \\
& \times\left(2^{2} \times 3^{2}\right)^{2}+(15 p q-13 p-13 q+11) \times\left(3^{2} \times 3^{2}\right)^{2} \\
= & 98415 p q-74413 p-74413 q+54140 .
\end{aligned}
$$

which is the required (24) result.

Theorem 6. Let $S i_{2} C_{3}-I I[p, q]$ be the silicon carbide. Then,

$$
\begin{aligned}
& S F\left(S i_{2} C_{3}-I I[p, q]\right)=\sqrt{2} \times\left(\frac{5 p q}{2}-\frac{13 p}{6}-\frac{13 q}{6}+\frac{11}{6}\right)+\sqrt{2} \times\left(\frac{p+q}{2}\right)+\sqrt{13} \times\left(\frac{8 p+8 q-14}{13}\right)+\frac{2 \times \sqrt{5}}{5}+\frac{\sqrt{10}}{10}, \\
& P F\left(S i_{2} C_{3}-I I[p, q]\right)=\frac{5 p q}{3}+\frac{7 p}{18}+\frac{7 q}{18}+\frac{2}{9} .
\end{aligned}
$$

Proof. Consider the graph silicon carbide $\mathrm{Si}_{2} \mathrm{C}_{3}-I I[p, q]$. By Remark 2, the graph $S_{2} C_{3}-I I[p, q]$ contains $10 p q$ vertices and $15 p q-2 p-3 q$ edges. By definition of the sum connectivity F-index of $\mathrm{Si}_{2} \mathrm{C}_{3}-I I[p, q]$, we have

$$
\begin{aligned}
S F\left(S i_{2} C_{3}-I I[p, q]\right)= & \sum_{u v \in E\left(S i_{2} C_{3}-I I[p, q]\right)} \frac{1}{\sqrt{\Gamma(u)^{2}+\Gamma(v)^{2}}} \\
= & \sum_{u v \in E_{1}\left(S i_{2} C_{3}-I I[p, q]\right)} \frac{1}{\sqrt{\Gamma(u)^{2}+\Gamma(v)^{2}}}+\sum_{u v \in E_{2}\left(S i_{2} C_{3}-I I[p, q]\right)} \frac{1}{\sqrt{\Gamma(u)^{2}+\Gamma(v)^{2}}} \\
& +\sum_{u v \in E_{3}\left(S i_{2} C_{3}-I I[p, q]\right)} \frac{1}{\sqrt{\Gamma(u)^{2}+\Gamma(v)^{2}}}+\sum_{u v \in E_{4}\left(S i_{2} C_{3}-I I[p, q]\right)} \frac{1}{\sqrt{\Gamma(u)^{2}+\Gamma(v)^{2}}} \\
& +\sum_{u v \in E_{5}\left(S i_{2} C_{3}-I I[p, q]\right)} \frac{1}{\sqrt{\Gamma(u)^{2}+\Gamma(v)^{2}}} \\
= & 2 \times \frac{1}{\sqrt{1^{2}+2^{2}}}+\frac{1}{\sqrt{1^{2}+3^{2}}}+(2 p+2 q) \times\left(\frac{1}{\sqrt{2^{2}+2^{2}}}\right)+(8 p+8 q-14) \\
& \times\left(\frac{1}{\sqrt{2^{2}+3^{2}}}\right)+(15 p q-13 p-13 q+11) \times\left(\frac{1}{\sqrt{3^{2}+3^{2}}}\right) \\
= & \sqrt{2} \times\left(\frac{5 p q}{2}-\frac{13 p}{6}-\frac{13 q}{6}+\frac{11}{6}\right)+\sqrt{2} \times\left(\frac{p+q}{2}\right) \\
& +\sqrt{13} \times\left(\frac{8 p+8 q-14}{13}\right)+\frac{2 \times \sqrt{5}}{5}+\frac{\sqrt{10}}{10} .
\end{aligned}
$$


which is the required (27) result.
By definition of product connectivity F-index of $\mathrm{Si}_{2} \mathrm{C}_{3}-I I[p, q]$, we have

$$
\begin{aligned}
& P F\left(S i_{2} C_{3}-I I[p, q]\right)=\sum_{u v \in E\left(S i_{2} C_{3}-I I[p, q]\right)} \frac{1}{\sqrt{\Gamma(u)^{2} \times \Gamma(v)^{2}}} \\
& =\sum_{u v \in E_{1}}\left(S_{\left.i_{2} C_{3}-I I[p, q]\right)} \frac{1}{\sqrt{\Gamma(u)^{2} \times \Gamma(v)^{2}}}+\sum_{u v \in E_{2}\left(S i_{2} C_{3}-I I[p, q]\right)} \frac{1}{\sqrt{\Gamma(u)^{2} \times \Gamma(v)^{2}}}\right.
\end{aligned}
$$

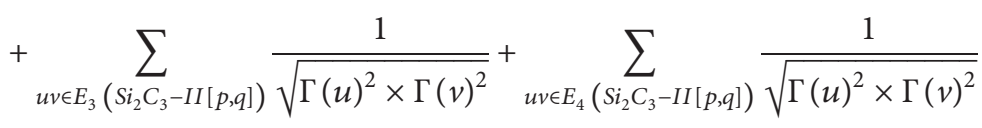

$$
\begin{aligned}
& +\sum_{u v \in E_{5}}\left(S_{\left.i_{2} C_{3}-I I[p, q]\right)} \frac{1}{\sqrt{\Gamma(u)^{2} \times \Gamma(v)^{2}}}\right. \\
& =2 \times \frac{1}{\sqrt{1^{2} \times 2^{2}}}+\frac{1}{\sqrt{1^{2} \times 3^{2}}}+(2 p+2 q) \times\left(\frac{1}{\sqrt{2^{2} \times 2^{2}}}\right)+(8 p+8 q-14) \\
& \times\left(\frac{1}{\sqrt{2^{2} \times 3^{2}}}\right)+(15 p q-13 p-13 q+11) \times\left(\frac{1}{\sqrt{3^{2} \times 3^{2}}}\right) \\
& =\frac{5 p q}{3}+\frac{7 p}{18}+\frac{7 q}{18}+\frac{2}{9}
\end{aligned}
$$

which is the required (28) result.

\section{Results for Silicon-Carbon $\mathrm{Si}_{2} \mathrm{C}_{3}-I I I[p, q]$}

In this section, we compute the generalization of Zagreb index, the generalized Zagreb index, the first and second hyper $F$-indices, the sum connectivity F-index, and the product connectivity F-index graphs of $\mathrm{Si}_{2} \mathrm{C}_{3}-\operatorname{III}[p, q]$. The $2 \mathrm{D}$ silicon-carbon $(\mathrm{Si}-\mathrm{C})$ single layers can be seen as configurable (or tunable) materials between the pure $2 D$ carbon single-layer graphene and the pure $2 D$ silicon singlelayer silicene. Lots of attempts have been conducted trying anticipating the most stable structures of the $\mathrm{SiC}$ sheet (for more details, see $[40,41])$.

The $2 D$ molecular graph of silicon carbide $\mathrm{Si}_{2} \mathrm{C}_{3}-I I I[p, q]$ is given in Figure 9, where carbon atom $C$ is shown in brown color and silicon atom $\mathrm{Si}$ is shown in blue color (for more details, see [42]). In Figure 10, we gave a demonstration how the cells connect in a row (chain) and how one row connects to another row; red lines (edges) show the connection between the unit cell in a chain and green lines (edges) connect the upper and lower rows (chains). We will denote this molecular graph by $S_{2} C_{3}-I I I[p, q]$.
Remark 3 (see [13]). The graph $\mathrm{Si}_{2} \mathrm{C}_{3}-I[p, q]$ contains $10 p q$ vertices and $15 p q-2 p-3 q$ edges.

We start by proving the carbon nanocones for the redefined Zagreb indices.

Theorem 7. Let $\mathrm{Si}_{2} \mathrm{C}_{3}-\mathrm{III}[p, q]$ be the silicon carbide. Then,

$$
\begin{aligned}
M_{\alpha, \beta}\left(S i_{2} C_{3}-I I I[p, q]\right)= & 9^{\alpha} \times \frac{(15 p q-10 p-13 q+8)}{6^{\beta}} \\
& +6^{\alpha} \times \frac{(8 p+8 q-12)}{5^{\beta}}+(4+4 q) \\
& \times 4^{\alpha-\beta}+\frac{2 \times 3^{\alpha}}{4^{\beta}}, \\
M_{r, s}\left(S i_{2} C_{3}-I I I[p, q]\right)= & (8 p+8 q-12) \times\left(2^{r} \times 3^{s}+2^{s}\right. \\
& \left.\times 3^{r}\right)+(30 p q-20 p-26 q+16) \\
& \times 3^{s+r}+(2+2 q) \times 4^{s+r}+2 \times 3^{r} \\
& +2 \times 3^{s} .
\end{aligned}
$$



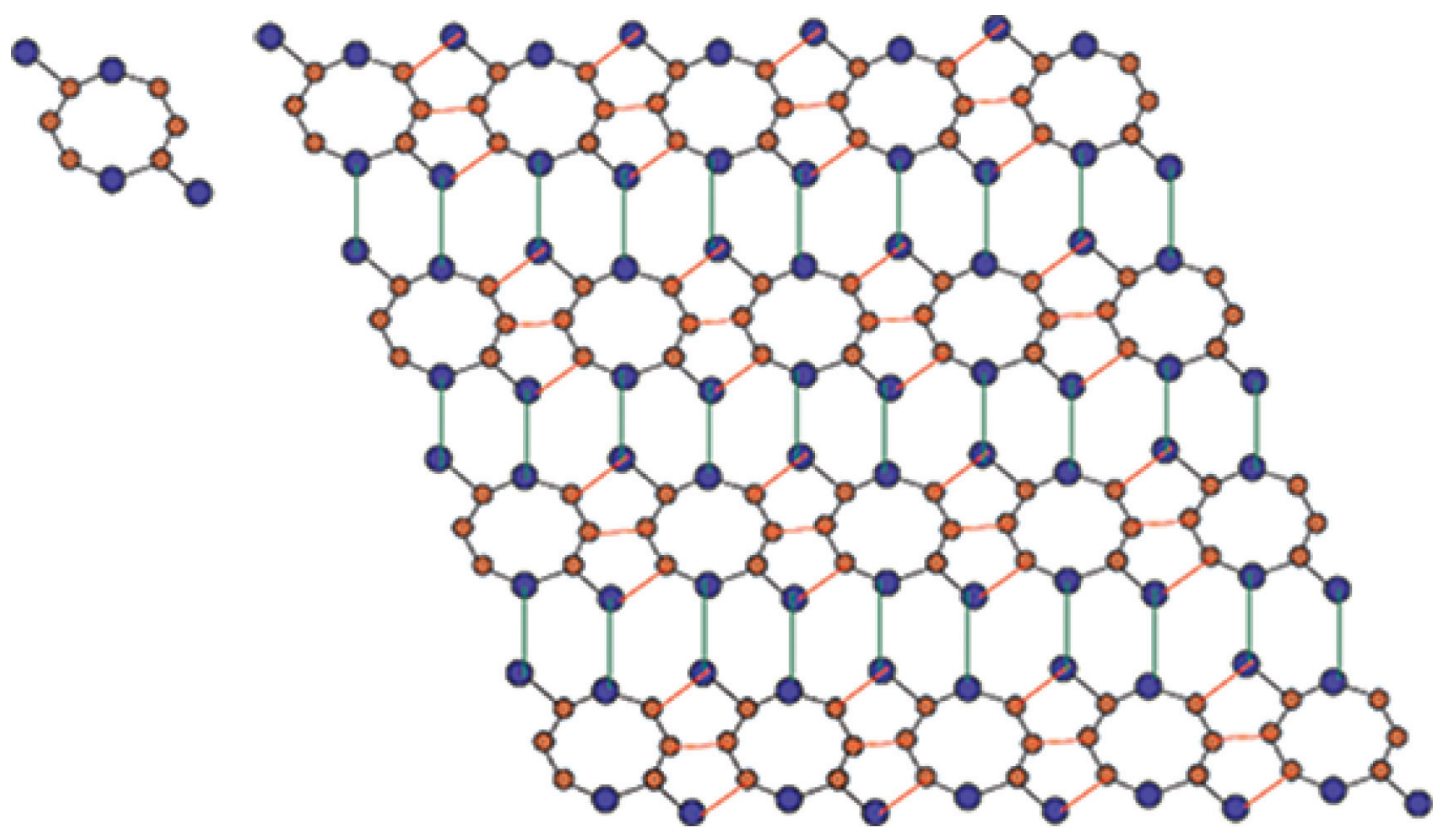

(a)

(b)

FIGURE 9: $2 \mathrm{D}$ structure of $S i_{2} C_{3}-I I I[p, q]$ : (a) unit cell of $S i_{2} C_{3}-I[p, q]$ and (b) $S i_{2} C_{3}-I[5,4]$.

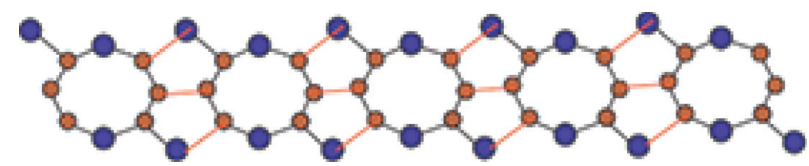

(a)

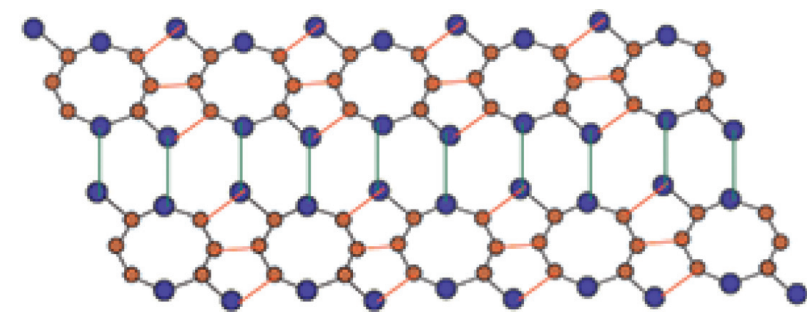

(b)

Figure 10: $2 \mathrm{D}$ structure of $S i_{2} C 3-I I I[p, q]:(\mathrm{a}) S i_{2} C_{3}-I[5,1]$ and (b) $S i_{2} C_{3}-I[5,2]$.

Proof. Consider the graph silicon carbide $\mathrm{Si}_{2} \mathrm{C}_{3}-\operatorname{III}[p, q]$. By Remark 3, the graph $\mathrm{Si}_{2} \mathrm{C}_{3}-I I I[p, q]$ contains $10 p q$ vertices and $15 p q-2 p-3 q$ edges. From the graph of $\mathrm{Si}_{2} \mathrm{C}_{3}-\mathrm{III}[p, q]$ silicon carbide, we can see that there are three partitions, $V_{\{1\}}=\left\{v \in V\left(S i_{2} C_{3}-I I I[p, q]\right)\right.$ $\mid \Gamma(v)=1\}, V_{\{2\}}=\left\{v \in V\left(S i_{2} C_{3}-I I I[p, q]\right) \mid \Gamma(v)=2\right\}$, and $V_{\{3\}}=\left\{v \in V\left(S i_{2} C_{3}-I I I[p, q]\right) \mid \Gamma(v)=3\right\}$. The edge set of the $\mathrm{Si}_{2} \mathrm{C}_{3}-I I I[p, q]$ can be partitioned as follows:

$$
\begin{aligned}
& E_{1}=\left\{e=u v \in E\left(S i_{2} C_{3}-I I I[p, q]\right) \mid \Gamma(u)=1 \text { and } \Gamma(v)=3\right\}, \\
& E_{2}=\left\{e=u v \in E\left(S i_{2} C_{3}-I I I[p, q]\right) \mid \Gamma(u)=2 \text { and } \Gamma(v)=2\right\}, \\
& E_{3}=\left\{e=u v \in E\left(S i_{2} C_{3}-I I I[p, q]\right) \mid \Gamma(u)=2 \text { and } \Gamma(v)=3\right\}, \\
& E_{4}=\left\{e=u v \in E\left(S i_{2} C_{3}-I I I[p, q]\right) \mid \Gamma(u)=3 \text { and } \Gamma(v)=3\right\} .
\end{aligned}
$$


From the molecular graph of $S_{2} C_{3}-I I I[p, q]$, we can observe that $\left|E_{1}\right|=2,\left|E_{2}\right|=p+2 q,\left|E_{3}\right|=8 p+8 q-12$, and $\left|E_{4}\right|=15 p q-10 p-13 q+8$.
Thus, by definition generalization of $\mathrm{Si}_{2} \mathrm{C}_{3}-\operatorname{III}[p, q]$, we have

$$
\begin{aligned}
M_{\alpha, \beta}\left(S i_{2} C_{3}-I I I[p, q]\right)= & \sum_{u v \in E\left(S i_{2} C_{3}-I I I[p, q]\right)} \frac{(\Gamma(u) \times \Gamma(v))^{\alpha}}{(\Gamma(u)+\Gamma(v))^{\beta}} \\
= & \sum_{u v \in E_{1}\left(S i_{2} C_{3}-I I I[p, q]\right)} \frac{(\Gamma(u) \times \Gamma(v))^{\alpha}}{(\Gamma(u)+\Gamma(v))^{\beta}}+\sum_{u v \in E_{2}\left(S i_{2} C_{3}-I I I[p, q]\right)} \frac{(\Gamma(u) \times \Gamma(v))^{\alpha}}{(\Gamma(u)+\Gamma(v))^{\beta}} \\
& +\sum_{u v \in E_{3}\left(S i_{2} C_{3}-I I I[p, q]\right)} \frac{(\Gamma(u) \times \Gamma(v))^{\alpha}}{(\Gamma(u)+\Gamma(v))^{\beta}}+\sum_{u v \in E_{4}\left(S i_{2} C_{3}-I I[p, q]\right)} \frac{(\Gamma(u) \times \Gamma(v))^{\alpha}}{(\Gamma(u)+\Gamma(v))^{\beta}} \\
= & 2 \times \frac{(1 \times 3)^{\alpha}}{(1+3)^{\beta}}+(p+2 q) \times \frac{(2 \times 2)^{\alpha}}{(2+2)^{\beta}} \\
& +(8 p+8 q-12) \times \frac{(2 \times 3)^{\alpha}}{(2+3)^{\beta}+(15 p q-10 p-13 q+8)} \\
& \times \frac{(3 \times 3)^{\alpha}}{(3+3)^{\beta}}=9^{\alpha} \times \frac{(15 p q-10 p-13 q+8)}{6^{\beta}}+6^{\alpha} \times \frac{(8 p+8 q-12)}{5^{\beta}} \\
& +(2+2 q) \times 4^{\alpha-\beta}+\frac{2 \times 3^{\alpha}}{4^{\beta}} .
\end{aligned}
$$

which is the required (31) result.

By definition of the generalized Zagreb index of $\mathrm{Si}_{2} \mathrm{C}_{3}-I I I[p, q]$, we have

$$
\begin{aligned}
M_{r, s}\left(S i_{2} C_{3}-I I I[p, q]\right)= & \sum_{u v \in E\left(S i_{2} C_{3}-I I I[p, q]\right)}\left(\Gamma(u)^{r} \times \Gamma(v)^{s}+\Gamma(u)^{s} \times \Gamma(v)^{r}\right) \\
= & \sum_{u v \in E_{1}\left(S i_{2} C_{3}-I I I[p, q]\right)}\left(\Gamma(u)^{r} \times \Gamma(v)^{s}+\Gamma(u)^{s} \times \Gamma(v)^{r}\right) \\
& +\sum_{u v \in E_{2}\left(S i_{2} C_{3}-I I I[p, q]\right)}\left(\Gamma(u)^{r} \times \Gamma(v)^{s}+\Gamma(u)^{s} \times \Gamma(v)^{r}\right) \\
& +\sum_{u v \in E_{3}\left(S i_{2} C_{3}-I I I[p, q]\right)}\left(\Gamma(u)^{r} \times \Gamma(v)^{s}+\Gamma(u)^{s} \times \Gamma(v)^{r}\right) \\
& +\sum_{u v \in E_{4}\left(S i_{2} C_{3}-I I I[p, q]\right)}\left(\Gamma(u)^{r} \times \Gamma(v)^{s}+\Gamma(u)^{s} \times \Gamma(v)^{r}\right) \\
= & 2 \times\left(1^{r} \times 3^{s}+1^{s} \times 3^{r}\right) \\
& +(p+2 q) \times\left(2^{r} \times 2^{s}+2^{s} \times 2^{r}\right)+(8 p+8 q-12) \\
& \times\left(2^{r} \times 3^{s}+2^{s} \times 3^{r}\right)+(15 p q-10 p-13 q+8) \times\left(3^{r} \times 3^{s}+3^{s} \times 3^{r}\right) \\
= & (8 p+8 q-12) \times\left(2^{r} \times 3^{s}+2^{s} \times 3^{r}\right)+(30 p q-20 p-26 q+16) \times 3^{s+r} \\
& +(4+4 q) \times 2^{s+r}+2 \times 3^{r}+2 \times 3^{s} .
\end{aligned}
$$


which is the required (32) result.

Theorem 8. Let $S i_{2} C_{3}-I I I[p, q]$ be the silicon carbide. Then,

$H F_{1}\left(S i_{2} C_{3}-I I I[p, q]\right)=4860 p q-2732 p-2732 q+1348$,

$\mathrm{HF}_{2}\left(\mathrm{Si}_{2} \mathrm{C}_{3}-\mathrm{III}[p, q]\right)=98415 p q-74413 p$

$-74413 q+54140$.
Proof. Consider the graph silicon carbide $\mathrm{Si}_{2} \mathrm{C}_{3}-I I I[p, q]$. By Remark 3, the graph $S i_{2} C_{3}-I I I[p, q]$ contains $10 p q$ vertices and $15 p q-2 p-3 q$ edges. By definition of the first hyper F-index of $\mathrm{Si}_{2} \mathrm{C}_{3}-I I I[p, q]$, we have

$$
\begin{aligned}
H F_{1}\left(S i_{2} C_{3}-I I I[p, q]\right)= & \sum_{u v \in E\left(S i_{2} C_{3}-I I I[p, q]\right)}\left(\Gamma(u)^{2}+\Gamma(v)^{2}\right)^{2} \\
= & \sum_{u v \in E_{1}\left(S i_{2} C_{3}-I I I[p, q]\right)}\left(\Gamma(u)^{2}+\Gamma(v)^{2}\right)^{2}+\sum_{u v \in E_{2}\left(S i_{2} C_{3}-I I I[p, q]\right)}\left(\Gamma(u)^{2}+\Gamma(v)^{2}\right)^{2} \\
& +\sum_{u v \in E_{3}\left(S i_{2} C_{3}-I I I[p, q]\right)}\left(\Gamma(u)^{2}+\Gamma(v)^{2}\right)^{2}+\sum_{u v \in E_{4}\left(S i_{2} C_{3}-I I I[p, q]\right)}\left(\Gamma(u)^{2}+\Gamma(v)^{2}\right)^{2} \\
= & 2 \times\left(1^{2}+3^{2}\right)^{2}+(2 p+2) \times\left(2^{2}+2^{2}\right)^{2}+(8 p+8 q-12) \\
& \times\left(2^{2}+3^{2}\right)^{2}+(15 p q-10 p-13 q+8) \times\left(3^{2}+3^{2}\right)^{2} \\
= & 4860 p q-1888 p-2732 q+892 .
\end{aligned}
$$

which is the required (36) result.

By definition of the second hyper F-index of $\mathrm{Si}_{2} \mathrm{C}_{3}-I I I[p, q]$, we have

$$
\begin{aligned}
H F_{2}\left(S i_{2} C_{3}-I I[p, q]\right)= & \sum_{u v \in E\left(S i_{2} C_{3}-I I I[p, q]\right)}\left(\Gamma(v)^{2} \times \Gamma(v)^{2}\right)^{2} \\
= & \sum_{u v \in E_{1}} \sum_{\left(S i_{2} C_{3}-I I I[p, q]\right)}\left(\Gamma(v)^{2} \times \Gamma(v)^{2}\right)^{2}+\sum_{u v \in E_{2}\left(S i_{2} C_{3}-I I I[p, q]\right)}\left(\Gamma(v)^{2} \times \Gamma(v)^{2}\right)^{2} \\
& +\sum_{u v \in E_{3}\left(S i_{2} C_{3}-I I I[p, q]\right)}\left(\Gamma(v)^{2} \times \Gamma(v)^{2}\right)^{2}+\sum_{u v \in E_{4}\left(S i_{2} C_{3}-I I I[p, q]\right)}\left(\Gamma(v)^{2} \times \Gamma(v)^{2}\right)^{2} \\
& +\sum_{u v \in E_{5}\left(S i_{2} C_{3}-I I I[p, q]\right)}\left(\Gamma(v)^{2} \times \Gamma(v)^{2}\right)^{2} \\
= & 2\left(1^{2} \times 3^{2}\right)^{2}+(2+2 q) \times\left(2^{2} \times 2^{2}\right)^{2}+(8 p+8 q-12) \\
& \times\left(2^{2} \times 3^{2}\right)^{2}+(15 p q-10 p-13 q+8) \times\left(3^{2} \times 3^{2}\right)^{2} \\
= & 98415 p q-54730 p-74413 q+37136 .
\end{aligned}
$$

which is the required (37) result.

Theorem 9. Let $S i_{2} C_{3}-I I I[p, q]$ be the silicon carbide. Then, 


$$
\begin{aligned}
& S F\left(S i_{2} C_{3}-I I I[p, q]\right)=\sqrt{2} \times\left(\frac{5 p q}{2}-\frac{5 p}{3}-\frac{13 q}{6}+\frac{4}{3}\right)+\sqrt{13} \times\left(\frac{(8 p+8 q-12)}{13}\right)+\sqrt{2} \times\left(\frac{q+1}{2}\right)+\frac{\sqrt{10}}{5}, \\
& P F\left(S i_{2} C_{3}-I I I[p, q]\right)=\frac{5 p q}{3}+\frac{2 p}{9}+\frac{7 q}{18}-\frac{5}{18} .
\end{aligned}
$$

Proof. Consider the graph silicon carbide $\mathrm{Si}_{2} \mathrm{C}_{3}-\operatorname{III}[p, q]$. By Remark 3, the graph $\mathrm{Si}_{2} \mathrm{C}_{3}-I I I[p, q]$ contains $10 p q$ vertices and $15 p q-2 p-3 q$ edges. By definition of the sum connectivity F-index of $\mathrm{Si}_{2} \mathrm{C}_{3}-I I I[p, q]$, we have

$$
\begin{aligned}
S F\left(S i_{2} C_{3}-I I I[p, q]\right)= & \sum_{u v \in E\left(S i_{2} C_{3}-I I I[p, q]\right)} \frac{1}{\sqrt{\Gamma(u)^{2}+\Gamma(v)^{2}}} \\
= & \sum_{u v \in E_{1}\left(S i_{2} C_{3}-I I I[p, q]\right)} \frac{1}{\sqrt{\Gamma(u)^{2}+\Gamma(v)^{2}}}+\sum_{u v \in E_{2}\left(S i_{2} C_{3}-I I I[p, q]\right)} \frac{1}{\sqrt{\Gamma(u)^{2}+\Gamma(v)^{2}}} \\
& +\sum_{u v \in E_{3}\left(S_{2} C_{3}-I I I[p, q]\right)} \frac{1}{\sqrt{\Gamma(u)^{2}+\Gamma(v)^{2}}}+\sum_{u v \in E_{4}\left(S i_{2} C_{3}-I I I[p, q]\right)} \frac{1}{\sqrt{\Gamma(u)^{2}+\Gamma(v)^{2}}} \\
= & 2 \times \frac{1}{\sqrt{1^{2}+3^{2}}}+(2+2 q) \times\left(\frac{1}{\sqrt{2^{2}+2^{2}}}\right)+(8 p+8 q-12) \\
& \times\left(\frac{1}{\sqrt{2^{2}+3^{2}}}\right)+(15 p q-10 p-13 q+8) \times\left(\frac{1}{\sqrt{3^{2}+3^{2}}}\right) \\
= & \sqrt{2} \times\left(\frac{5 p q}{2}-\frac{5 p}{3}-\frac{13 q}{6}+\frac{4}{3}\right)+\sqrt{13} \times\left(\frac{(8 p+8 q-12)}{13}\right) \\
& +\sqrt{2} \times\left(\frac{q+1}{2}\right)+\frac{\sqrt{10}}{5} .
\end{aligned}
$$

which is the required (40) result.

By definition of product connectivity F-index of $\mathrm{Si}_{2} \mathrm{C}_{3}-\operatorname{III}[p, q]$, we have

$$
\begin{aligned}
P F\left(S i_{2} C_{3}-I I I[p, q]\right)= & \sum_{u v \in E\left(S i_{2} C_{3}-I I I[p, q]\right)} \frac{1}{\sqrt{\Gamma(u)^{2} \times \Gamma(v)^{2}}} \\
= & \sum_{u v \in E_{1}\left(S i_{2} C_{3}-I I I[p, q]\right)} \frac{1}{\sqrt{\Gamma(u)^{2} \times \Gamma(v)^{2}}}+\sum_{u v \in E_{2}\left(S i_{2} C_{3}-I I I[p, q]\right)} \frac{1}{\sqrt{\Gamma(u)^{2} \times \Gamma(v)^{2}}} \\
& +\sum_{u v \in E_{3}\left(S i_{2} C_{3}-I I I[p, q]\right)} \frac{1}{\sqrt{\Gamma(u)^{2} \times \Gamma(v)^{2}}}+\sum_{u v \in E_{4}\left(S i_{2} C_{3}-I I I[p, q]\right)} \frac{1}{\sqrt{\Gamma(u)^{2} \times \Gamma(v)^{2}}} \\
= & 2 \times \frac{1}{\sqrt{1^{2} \times 3^{2}}}+(2+2 q) \times\left(\frac{1}{\sqrt{2^{2} \times 2^{2}}}\right)+(8 p+8 q-12) \\
& \times\left(\frac{1}{\sqrt{2^{2} \times 3^{2}}}\right)+(15 p q-10 p-13 q+8) \times\left(\frac{1}{\sqrt{3^{2} \times 3^{2}}}\right) \\
= & \frac{5 p q}{3}+\frac{2 p}{9}+\frac{7 q}{18}-\frac{5}{18} .
\end{aligned}
$$




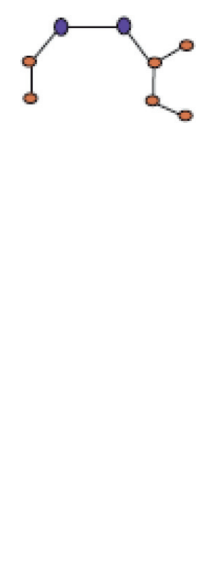

(a)

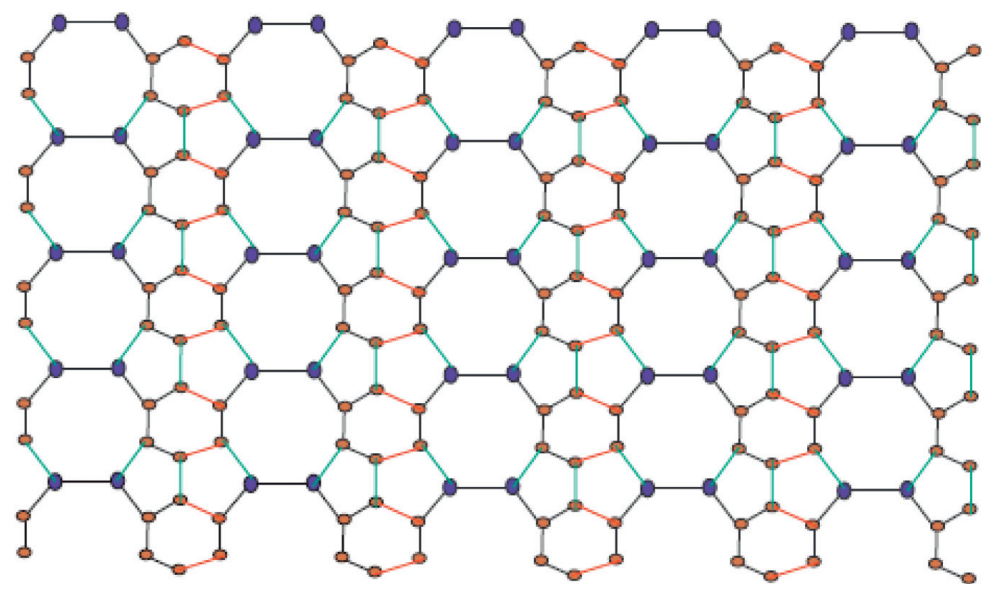

(b)

Figure 11: $2 \mathrm{D}$ structure of $\mathrm{SiC}_{3}-I I I[p, q]$ : (a) $\mathrm{SiC}_{3}-I[5,1]$ and (b) $\mathrm{SiC}_{3}-I[5,2]$.

which is the required (41) result.

\section{Results for Silicon-Carbon $\mathrm{Si}_{2} \mathrm{C}_{3}-\operatorname{III}[p, q]$}

In this section, we compute the generalization of Zagreb index, the generalized Zagreb index, the first and second hyper $F$-indices, the sum connectivity $\mathrm{F}$-index, and the product connectivity F-index graphs of $\mathrm{Si}_{2} \mathrm{C}_{3}-I I I[p, q]$.

The $2 \mathrm{D}$ molecular graph of silicon carbide $\mathrm{SiC}_{3}-I I I$ is given in Figure 11, where carbon atom $C$ is shown in brown color and silicon atom $\mathrm{Si}$ is shown in blue color (for more details, see [42]). In Figure 12, we gave a demonstration how the cells connect in a row (chain) and how one row connects to another row; red lines show the connection between the unit cells and green lines (edges) connect the upper and lower rows. We will denote this molecular graph by $\mathrm{Si}_{2} \mathrm{C}_{3}-I I I[p, q]$.

Remark 4 (see [13]). The graph $\mathrm{Si}_{2} \mathrm{C}_{3}-I I I[p, q]$ contains $8 p q$ vertices and $12 p q-3 p-2 q$. edges.

We start by proving the carbon nanocones for the redefined Zagreb indices.

Theorem 10. Let $\mathrm{Si}_{2} \mathrm{C}_{3}-I I I[p, q]$ be the silicon carbide. Then,

$$
\begin{aligned}
M_{\alpha, \beta}\left(\mathrm{SiC}_{3}-I I I[p, q]\right)= & 9^{\alpha} \times \frac{(12 p q-12 p-8 q+8)}{6^{\beta}}+6^{\alpha} \\
& \times \frac{(6 p+4 q-8)}{5^{\beta}}+(3 p+2 q-3) \\
& \times 4^{\alpha-\beta}+\frac{3^{\alpha}}{4^{\beta}}+\frac{2 \times 2^{\alpha}}{3^{\beta}}
\end{aligned}
$$

$$
\begin{aligned}
M_{r, s}\left(\mathrm{SiC}_{3}-I I I[p, q]\right)= & (6 p+4 q-8) \times\left(2^{r} \times 3^{s}+2^{s}\right. \\
& \left.\times 3^{r}\right)+(24 p q-24 p-16 q+16) \\
& \times 3^{s+r}+(6 p+4 q-6) \times 2^{s+r}+3^{r} \\
& +3^{s}+2 \times 2^{r}+2 \times 2^{s} .
\end{aligned}
$$

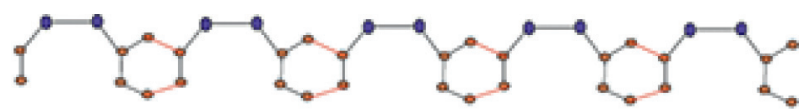

(a)

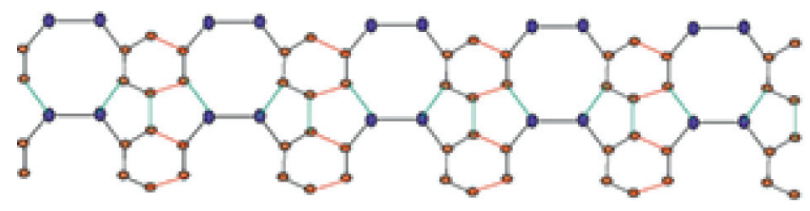

(b)

Figure 12: $2 D$ structure of $\mathrm{SiC}_{3}-I I I[p, q]$ : (a) $\mathrm{SiC}_{3}-I[5,1]$ and (b) $\mathrm{SiC}_{3}-I[5,2]$.

Proof. Consider the graph silicon carbide $\mathrm{Si}_{2} \mathrm{C}_{3}-I I I[p, q]$. By Remark 4, the graph $S i_{2} C_{3}-I I I[p, q]$ contains $8 p q$ vertices and $12 p q-3 p-2 q$ edges. From the graph of $\mathrm{Si}_{2} \mathrm{C}_{3}-I I I[p, q]$ silicon carbide, we can see that there are three partitions, $V_{\{1\}}=\left\{v \in V\left(\operatorname{SiC}_{3}-I I I[p, q]\right) \mid \Gamma(v)=1\right\}$, $V_{\{2\}}=\left\{v \in V\left(\mathrm{SiC}_{3}-I I I[p, q]\right) \mid \Gamma(v)=2\right\}$, and $V_{\{3\}}=\{v \in$ $\left.V\left(\mathrm{SiC}_{3}-I I I[p, q]\right) \mid \Gamma(v)=3\right\}$. The edge set of the $\mathrm{Si}_{2} \mathrm{C}_{3}-$ $I I I[p, q]$ can be partitioned as follows:

$$
\begin{aligned}
& E_{1}=\left\{e=u v \in E\left(\operatorname{SiC}_{3}-I I I[[p, q]) \mid \Gamma(u)=1 \text { and } \Gamma(v)=2\right\},\right. \\
& E_{2}=\left\{e=u v \in E\left(\operatorname{SiC}_{3}-I I I[[p, q]) \mid \Gamma(u)=1 \text { and } \Gamma(v)=3\right\},\right. \\
& E_{3}=\left\{e=u v \in E\left(\operatorname{SiC}_{3}-I I I[[p, q]) \mid \Gamma(u)=2 \text { and } \Gamma(v)=2\right\},\right. \\
& E_{4}=\left\{e=u v \in E\left(\operatorname{SiC}_{3}-I I I[[p, q]) \mid \Gamma(u)=2 \text { and } \Gamma(v)=3\right\},\right. \\
& E_{1}=\left\{e=u v \in E\left(\operatorname{SiC}_{3}-I I I[[p, q]) \mid \Gamma(u)=3 \text { and } \Gamma(v)=3\right\} .\right.
\end{aligned}
$$

From the molecular graph of $\mathrm{Si}_{2} \mathrm{C}_{3}-I I I[p, q]$, we can observe that $\left|E_{1}\right|=2,\left|E_{2}\right|=1,\left|E_{3}\right|=3 p+2 q-3,\left|E_{4}\right|=$ $6 p+4 q-8$, and $\left|E_{5}\right|=(12 p q-12 p-8 q+8)$.

Thus, by definition generalization of $\mathrm{Si}_{2} \mathrm{C}_{3}-I I I[p, q]$, we have 


$$
\begin{aligned}
M_{\alpha, \beta}\left(S_{i} C_{3}-I I I[p, q]\right)= & \sum_{u v \in E\left(S i C_{3}-I I I[p, q]\right)} \frac{(\Gamma(u) \times \Gamma(v))^{\alpha}}{(\Gamma(u)+\Gamma(v))^{\beta}} \\
= & \sum_{\left.u v \in E_{1}\left(S i C_{3}-I I I p, q\right]\right)} \frac{(\Gamma(u) \times \Gamma(v))^{\alpha}}{(\Gamma(u)+\Gamma(v))^{\beta}}+\sum_{u v \in E_{2}\left(S i C_{3}-I I I[p, q]\right)} \frac{(\Gamma(u) \times \Gamma(v))^{\alpha}}{(\Gamma(u)+\Gamma(v))^{\beta}} \\
& +\sum_{u v \in E_{3}\left(S i C_{3}-I I I[p, q]\right)} \frac{(\Gamma(u) \times \Gamma(v))^{\alpha}}{(\Gamma(u)+\Gamma(v))^{\beta}}+\sum_{u v \in E_{4}\left(S i C_{3}-I I I[p, q]\right)} \frac{(\Gamma(u) \times \Gamma(v))^{\alpha}}{(\Gamma(u)+\Gamma(v))^{\beta}} \\
& +\sum_{u v \in E_{5}\left(S i C_{3}-I I I[p, q]\right)} \frac{(\Gamma(u) \times \Gamma(v))^{\alpha}}{(\Gamma(u)+\Gamma(v))^{\beta}} \\
= & 2 \times \frac{(1 \times 2)^{\alpha}}{(1+2)^{\beta}}+\frac{(1 \times 3)^{\alpha}}{(1+3)^{\beta}+(3 p+2 q-3) \times \frac{(2 \times 2)^{\alpha}}{(2+2)^{\beta}}} \\
& +(6 p+4 q-8) \times \frac{(2 \times 3)^{\alpha}}{(2+3)^{\beta}+(12 p q-12 p-8 q+8)} \\
& \times \frac{(3 \times 3)^{\alpha}}{(3+3)^{\beta}}=9^{\alpha} \times \frac{(12 p q-12 p-8 q+8)}{6^{\beta}}+6^{\alpha} \times \frac{(6 p+4 q-8)}{5^{\beta}} \\
& +(3 p+2 q-3) \times 4^{\alpha-\beta}+\frac{3^{\alpha}}{4^{\beta}}+\frac{2 \times 2^{\alpha}}{3^{\beta}} .
\end{aligned}
$$

which is the required (44) result.

By definition of the generalized Zagreb index of $\mathrm{SiC}_{3}-\mathrm{III}[p, q]$, we have

$$
\begin{aligned}
& M_{r, s}\left(S i C_{3}-I I I[p, q]\right)=\sum_{u v \in E\left(S i C_{3}-I I I[p, q]\right)}\left(\Gamma(u)^{r} \times \Gamma(v)^{s}+\Gamma(u)^{s} \times \Gamma(v)^{r}\right) \\
& =\sum_{u v \in E_{1}\left(S i C_{3}-I I I[p, q]\right)}\left(\Gamma(u)^{r} \times \Gamma(v)^{s}+\Gamma(u)^{s} \times \Gamma(v)^{r}\right) \\
& +\sum_{u v \in E_{2}\left(S i C_{3}-I I I[p, q]\right)}\left(\Gamma(u)^{r} \times \Gamma(v)^{s}+\Gamma(u)^{s} \times \Gamma(v)^{r}\right) \\
& +\sum_{u v \in E_{3}\left(S i C_{3}-I I I[p, q]\right)}\left(\Gamma(u)^{r} \times \Gamma(v)^{s}+\Gamma(u)^{s} \times \Gamma(v)^{r}\right) \\
& +\sum_{u v \in E_{4}}\left(\sum_{\left.i C_{3}-I I I[p, q]\right)}\left(\Gamma(u)^{r} \times \Gamma(v)^{s}+\Gamma(u)^{s} \times \Gamma(v)^{r}\right)\right. \\
& +\sum_{u v \in E_{5}}\left(\sum_{\left(i C_{3}-I I I[p, q]\right)}\left(\Gamma(u)^{r} \times \Gamma(v)^{s}+\Gamma(u)^{s} \times \Gamma(v)^{r}\right)\right. \\
& =2 \times\left(1^{r} \times 2^{s}+1^{s} \times 2^{r}\right)+\left(1^{r} \times 3^{s}+1^{s} \times 3^{r}\right) \\
& +(3 p+2 q-3) \times\left(2^{r} \times 2^{s}+2^{s} \times 2^{r}\right)+(6 p+4 q-8) \\
& \times\left(2^{r} \times 3^{s}+2^{s} \times 3^{r}\right)+(12 p q-12 p-8 q+8) \times\left(3^{r} \times 3^{s}+3^{s} \times 3^{r}\right) \\
& =(6 p+4 q-8) \times\left(2^{r} \times 3^{s}+2^{s} \times 3^{r}\right)+(24 p q-24 p-16 q+16) \times 3^{s+r} \\
& +(6 p+4 q-6) \times 2^{s+r}+3^{r}+3^{s}+2 \times 2^{r}+2 \times 2^{s} .
\end{aligned}
$$


which is the required (45) result.

Theorem 11. Let $\mathrm{SiC}_{3}-\operatorname{III}[p, q]$ be the silicon carbide. Then,

$H F_{1}\left(\operatorname{SiC}_{3}-I I I[p, q]\right)=3888 p q-2682 p-1788 q+1198$,
Proof. Consider the graph silicon carbide $\mathrm{SiC}_{3}-\mathrm{III}[p, q]$. By Remark 4, the graph $\operatorname{SiC}_{3}-\operatorname{III}[p, q]$ contains $8 p q$ vertices and $12 p q-3 p-2 q$. edges. By definition of the first hyper F-index of $\mathrm{SiC}_{3}-I I I[p, q]$, we have

$$
\begin{aligned}
H F_{1}\left(S i C_{3}-I I I[p, q]\right)= & \sum_{u v \in E\left(S i C_{3}-I I I[p, q]\right)}\left(\Gamma(u)^{2}+\Gamma(v)^{2}\right)^{2} \\
= & \sum_{u v \in E_{1}} \sum_{\left(S i C_{3}-I I I[p, q]\right)}\left(\Gamma(u)^{2}+\Gamma(v)^{2}\right)^{2}+\sum_{u v \in E_{2}\left(S i C_{3}-I I I[p, q]\right)}\left(\Gamma(u)^{2}+\Gamma(v)^{2}\right)^{2} \\
& +\sum_{u v \in E_{3}} \sum_{\left.S i C_{3}-I I I[p, q]\right)}\left(\Gamma(u)^{2}+\Gamma(v)^{2}\right)^{2}+\sum_{u v \in E_{4}\left(S i C_{3}-I I I[p, q]\right)}\left(\Gamma(u)^{2}+\Gamma(v)^{2}\right)^{2} \\
& +\sum_{u v \in E_{5}\left(S i C_{3}-I I I[p, q]\right)}\left(\Gamma(u)^{2}+\Gamma(v)^{2}\right)^{2} \\
= & 2 \times\left(1^{2}+2^{2}\right)^{2}+\left(1^{2}+3^{2}\right)^{2}+(3 p+2 q-3) \times\left(2^{2}+2^{2}\right)^{2} \\
& +(6 p+4 q-8) \times\left(2^{2}+3^{2}\right)^{2}+(12 p q-12 p-8 q+8) \times\left(3^{2}+3^{2}\right)^{2} \\
= & 3888 p q-2682 p-1788 q+1198 .
\end{aligned}
$$

which is the required (49) result.

By definition of the second hyper F-index of $\mathrm{Si}_{2} \mathrm{C}_{3}-I I I[p, q]$, we have

$$
\begin{aligned}
H F_{2}\left(S i C_{3}-I I I[p, q]\right)= & \sum_{u v \in E\left(S i C_{3}-I I I[p, q]\right)}\left(\Gamma(u)^{2} \times \Gamma(v)^{2}\right)^{2} \\
= & \sum_{u v \in E_{1}\left(S_{\left.i C_{3}-I I I[p, q]\right)}\left(\Gamma(u)^{2} \times \Gamma(v)^{2}\right)^{2}+\sum_{u v \in E_{2}\left(S i C_{3}-I I I[p, q]\right)}\left(\Gamma(u)^{2} \times \Gamma(v)^{2}\right)^{2}\right.} \\
& +\sum_{u v \in E_{3}\left(S i C_{3}-I I I[p, q]\right)}\left(\Gamma(u)^{2} \times \Gamma(v)^{2}\right)^{2}+\sum_{u v \in E_{4}\left(S i C_{3}-I I I[p, q]\right)}\left(\Gamma(u)^{2} \times \Gamma(v)^{2}\right)^{2} \\
& +\sum_{u v \in E_{5}\left(S i C_{3}-I I I[p, q]\right)}\left(\Gamma(u)^{2} \times \Gamma(v)^{2}\right)^{2} \\
= & 2 \times\left(1^{2} \times 2^{2}\right)^{2}+\left(1^{2} \times 3^{2}\right)^{2}+(3 p+2 q-3) \times\left(2^{2} \times 2^{2}\right)^{2} \\
& +(6 p+4 q-8) \times\left(2^{2} \times 3^{2}\right)^{2}+(12 p q-12 p-8 q+8) \times\left(3^{2} \times 3^{2}\right)^{2} \\
= & 78732 p q-70188 p-46792 q+41465 .
\end{aligned}
$$


which is the required (50) result.

Theorem 12. Let $\mathrm{SiC}_{3}-I I I[p, q]$ be the silicon carbide. Then,

$$
\begin{aligned}
S F\left(S i C_{3}-I I I[p, q]\right)= & \sqrt{2} \times\left(2 p q-\frac{4 p}{3}-2 p+\frac{4}{3}\right)+\sqrt{13} \\
& \times\left(\frac{(6 p+4 q-8)}{13}\right)+\sqrt{2} \\
& \times\left(\frac{q}{2}+\frac{3 q}{2}-\frac{3}{4}\right)+\frac{\sqrt{10}}{10}+\frac{2 \sqrt{5}}{5} \\
\operatorname{PF}\left(\mathrm{SiC}_{3}-I I I[p, q]\right) & =\frac{4 p q}{3}+\frac{5 p}{12}+\frac{5 p}{18}+\frac{5}{36} .
\end{aligned}
$$

Proof. Consider the graph silicon carbide $\mathrm{SiC}_{3}-I I I[p, q]$. By Remark 4, the graph $\mathrm{SiC}_{3}-I I I[p, q]$ contains $8 p q$ vertices and $12 p q-3 p-2 q$ edges. By definition of the sum connectivity F-index of $\mathrm{SiC}_{3}-I I I[p, q]$, we have

$$
\begin{aligned}
& S F\left(\operatorname{SiC}_{3}-I I I[p, q]\right)=\sum_{u v \in E\left(S i C_{3}-I I I[p, q]\right)} \frac{1}{\sqrt{\Gamma(u)^{2}+\Gamma(v)^{2}}} \\
& =\sum_{u v \in E_{1}} \frac{1}{\left(S i C_{3}-I I I[p, q]\right)} \frac{1}{\sqrt{\Gamma(u)^{2}+\Gamma(v)^{2}}}+\sum_{u v \in E_{2}} \sum_{\left(S i C_{3}-I I I[p, q]\right)} \frac{1}{\sqrt{\Gamma(u)^{2}+\Gamma(v)^{2}}} \\
& +\sum_{u v \in E_{3}} \frac{1}{\left(S i C_{3}-I I I[p, q]\right)} \frac{1}{\sqrt{\Gamma(u)^{2}+\Gamma(v)^{2}}}+\sum_{u v \in E_{4}\left(S i C_{3}-I I I[p, q]\right)} \frac{1}{\sqrt{\Gamma(u)^{2}+\Gamma(v)^{2}}} \\
& +\sum_{u v \in E_{5}} \frac{1}{\left(S i C_{3}-I I I[p, q]\right)} \frac{1}{\sqrt{\Gamma(u)^{2}+\Gamma(v)^{2}}} \\
& =2 \times \frac{1}{\sqrt{1^{2}+2^{2}}}+\frac{1}{\sqrt{1^{2}+3^{2}}}+(3 p+2 q-3) \times\left(\frac{1}{\sqrt{2^{2}+2^{2}}}\right)+(6 p+4 q-8) \\
& \times\left(\frac{1}{\sqrt{2^{2}+3^{2}}}\right)+(12 p q-12 p-8 q+8) \times\left(\frac{1}{\sqrt{3^{2}+3^{2}}}\right) \\
& =\sqrt{2} \times\left(2 p q-\frac{4 p}{3}-2 p+\frac{4}{3}\right)+\sqrt{13} \times\left(\frac{(6 p+4 q-8)}{13}\right) \\
& +\sqrt{2} \times\left(\frac{q}{2}+\frac{3 q}{2}-\frac{3}{4}\right)+\frac{\sqrt{10}}{10}+\frac{2 \sqrt{5}}{5} .
\end{aligned}
$$


which is the required (53) result.

By definition of product connectivity F-index of $\mathrm{Si}_{2} \mathrm{C}_{3}-I I I[p, q]$, we have

$$
\begin{aligned}
P F\left(S i C_{3}-I I I[p, q]\right)= & \sum_{u v \in E\left(S i C_{3}-I I I[p, q]\right)} \frac{1}{\sqrt{\Gamma(u)^{2} \times \Gamma(v)^{2}}} \\
= & \sum_{u v \in E_{1}\left(S i C_{3}-I I I[p, q]\right)} \frac{1}{\sqrt{\Gamma(u)^{2} \times \Gamma(v)^{2}}}+\sum_{u v \in E_{2}\left(S i C_{3}-I I I[p, q]\right)} \frac{1}{\sqrt{\Gamma(u)^{2} \times \Gamma(v)^{2}}} \\
& +\sum_{u v \in E_{3}\left(S i C_{3}-I I I[p, q]\right)} \frac{1}{\sqrt{\Gamma(u)^{2} \times \Gamma(v)^{2}}} \\
& +\sum_{u v \in E_{4}\left(S i C_{3}-I I I[p, q]\right)} \underbrace{\sqrt{\Gamma(u)^{2} \times \Gamma(v)^{2}}}+\sum_{u v \in E_{5}\left(S i C_{3}-I I I[p, q]\right)} \frac{1}{\sqrt{\Gamma(u)^{2} \times \Gamma(v)^{2}}} \\
= & 2 \times \frac{1}{\sqrt{1^{2} \times 2^{2}}}+\frac{1}{\sqrt{1^{2} \times 3^{2}}+(3 p+2 q-3) \times\left(\frac{1}{\sqrt{2^{2} \times 2^{2}}}\right)+(6 p+4 q-8)} \\
& \times\left(\frac{1}{\sqrt{2^{2} \times 3^{2}}}\right)+(12 p q-12 p-8 q+8) \times\left(\frac{1}{\sqrt{3^{2} \times 3^{2}}}\right) \\
= & \frac{4 p q}{3}+\frac{5 p}{12}+\frac{5 p}{18}+\frac{5}{36} .
\end{aligned}
$$

which is the required (54) result.

\section{Conclusion}

In this paper, we computed the generalization of Zagreb index, the generalized Zagreb index, the first and second hyper F-indices, the sum connectivity F-index, and the product connectivity F-index graphs of $S i_{2} C_{3}-I[p, q], S i_{2} C_{3}-I I[p, q], \quad S i_{2} C_{3}-I I I[p, q] \quad$ and $\mathrm{Si}_{2} \mathrm{C}_{3}-I I I[p, q]$.

\section{Data Availability}

The data used to support the findings of this study are cited at relevant places within the text as references.

\section{Conflicts of Interest}

The authors declare that there no conflicts of interest.

\section{Authors' Contributions}

All authors contributed equally to this work.

\section{Acknowledgments}

This research was supported by Quality Engineering Projects of Anhui University (grant no. 2018jyxm1074) and Natural Science Fund of Education Department of Anhui Province (grant no. KJ2018A0598).

\section{References}

[1] M. Saheli, H. Saati, and A. R. Ashrafi, "The eccentric connectivity index of one pentagonal carbon nanocones," Optoelectronics and Advanced Materials: Rapid Communications, vol. 4, p. 896, 2010.

[2] N. Trinajstić, Chemical Graph Theory, CRC Press, Boca Raton, FL, USA, 1992

[3] C. Wang, S. Wang, and B. Wei, "Cacti with extremal PI index," Transactions on Combinatorics, vol. 5, no. 4, pp. 1-8, 2016.

[4] D. B. West, An Introduction to Graph Theory, Prentice-Hall, Upper Saddle River, NJ, USA, 1996.

[5] B. Zhao, J. Gan, and H. Wu, "Redefined Zagreb indices of some nano structures," Applied Mathematics and Nonlinear Sciences, vol. 1, no. 1, pp. 291-300, 2016.

[6] J.-B. Liu, J. Zhao, J. Min, and J. Cao, "The Hosoya index of graphs formed by a fractal graph," Fractals, vol. 27, no. 8, Article ID 1950135, 2019.

[7] J.-B. Liu, J. Zhao, and Z.-Q. Cai, "On the generalized adjacency, Laplacian and signless Laplacian spectra of the weighted edge corona networks," Physica A: Statistical Mechanics and its Applications, vol. 540, Article ID 123073, 2020.

[8] J.-B. Liu, J. Zhao, H. He, and Z. Shao, "Valency-based topological descriptors and structural property of the generalized Sierpiński networks," Journal of Statistical Physics, vol. 177, no. 6, pp. 1131-1147, 2019.

[9] S. C. Basak, D. Mills, M. M. Mumtaz, and K. Balasubramanian, "Use of topological indices in predicting aryl hydrocarbon receptor binding potency of dibenzofurans: a hierarchical QSAR approach," Indian Journal of Chemistry, vol. 42A, pp. 1385-1391, 2003. 
[10] I. García, Y. Fall, and G. Gómez, "Using topological indices to predict anti-Alzheimer and anti-parasitic GSK-3 inhibitors by multi-target QSAR in silico screening," Molecules, vol. 15, no. 8, pp. 5408-5422, 2010.

[11] M. Husin, R. Hasni, N. Arif, and M. Imran, "On topological indices of certain families of nanostar dendrimers," Molecules, vol. 21 , no. 7, p. 821, 2016.

[12] M. Naeem, M. K. Siddiqui, Sh. Qaisar, M. Imran, and M. R. Farahani, "Computing topological indices of 2-dimensional silicon-carbons," UPB Scientific Bulletin, Series B, vol. 80, pp. 115-136, 2018.

[13] Y. Kwun, A. Virk, W. Nazeer, M. Rehman, and S. Kang, "On the multiplicative degree-based topological indices of siliconcarbon $\mathrm{Si}_{2} \mathrm{C}_{3}-\mathrm{I}[p, q]$ and $\mathrm{Si}_{2} \mathrm{C}_{3}-\mathrm{II}[p, q]$," Symmetry, vol. 10, no. 8 , p. $320,2018$.

[14] M. Imran, M. K. Siddiqui, M. Naeem, and M. A. Iqbal, "On topological properties of symmetric chemical structures," Symmetry, vol. 10, 2018.

[15] N. Idrees, M. N. Naeem, F. Hussain, A. Sadiq, and M. K. Siddiqui, "Molecular descriptors of benzenoid system," Quimica Nova, vol. 40, pp. 143-145, 2017.

[16] V. R. Kulli, "F-indices of chemical networks," International Journal of Mathematical Archive, vol. 10, pp. 21-30, 2019.

[17] W. Gao, M. R. Farahani, M. K. Jamil, and M. K. Siddiqui, "The redefined first, second and third Zagreb indices of titania nanotubes $\mathrm{TiO}_{2}[m, n]$," The Open Biotechnology Journal, vol. 10, no. 1, pp. 272-277, 2016.

[18] S. M. Kang, M. K. Siddiqui, N. A. Rehman, M. Naeem, and M. H. Muhammad, "Topological properties of 2-dimensional silicon-carbons," IEEE Access, vol. 6, pp. 59362-59373, 2018.

[19] M. Ghorbani and M. Jalali, "The vertex PI, Szeged and omega polynomials of carbon Nanocones $\mathrm{CNC}_{4}[n]$." MATCH Communication in Mathematical and in Computer Chemistry, vol. 62, pp. 353-362, 2009.

[20] J. Gillot, W. Bollmann, and B. Lux, "Cristaux de graphite en forme de cigare et a structure conique," Carbon, vol. 6, no. 3, pp. 381-387, 1968.

[21] I. Gutman, "An exceptional property of the first Zagreb index," MATCH Communication in Mathematical and in Computer Chemistry, vol. 72, pp. 733-740, 2014.

[22] M. Khalifeh, H. Yousefi-Azari, and A. R. Ashrafi, "A method for computing the wiener index of one-pentagonal carbon nanocones," Current Nanoscience, vol. 6, no. 2, pp. 155-157, 2010.

[23] R. Todeschini and V. Consonni, Handbook of Molecular Descriptors, Wiley, Weinheim, Germany, 2000.

[24] D. B. West, Introduction to Graph Theory, vol. 2, PrenticeHall, Upper Saddle River, NJ, USA, 2001.

[25] I. Gutmana, E. Milovanović, and I. Milovanović, "Beyond the Zagreb indices," AKCE International Journal of Graphs and Combinatorics, In press, 2019.

[26] V. R. Kuli, "The gourava indices and coindices of graphs," Annals of Pure and Applied Mathematics, vol. 14, pp. 33-38, 2017.

[27] M. Azari and A. Ironmanesh, "Generalized Zagreb index of graphs," Studia Universitatis Babeş-Bolyai Mathematica, vol. 56, pp. 59-70, 2011.

[28] S. Ghobadi and M. Ghorbaninejad, "On F-polynomial, multiple and hyper F-index of some molecular graphs," Bulletin of Mathematical Sciences and Applications, vol. 20, pp. 36-43, 2018.

[29] T. Reti, A. Ali, P. Varga, and E. Bitay, "Some properties of the neighborhood first Zagreb index," Discrete Mathematics Letters, vol. 2, pp. 10-17, 2019.
[30] K. C. Das and A. Ali, "On a conjecture about the second Zagreb index," Discrete Mathematics Letters, vol. 2, pp. 38-43, 2019.

[31] S. M. Hosamani, B. Kulkarni, R. G. Boli, and V. M. Gadag, "QSPR analysis of certain graph theocratical matrices and their corresponding energy," Applied Mathematics and Nonlinear Sciences, vol. 2, no. 1, p. 131150, 2017.

[32] Z. Shao, M. Siddiqui, and M. Muhammad, "Computing Zagreb indices and Zagreb polynomials for symmetrical nanotubes," Symmetry, vol. 10, no. 7, pp. 244-254, 2018.

[33] Z. Shao, P. Wu, X. Zhang, D. Dimitrov, and J. B. Liu, "On the maximum $A B C$ index of graphs with prescribed size and without pendent vertices," IEEE Access, vol. 6, pp. 604-616, 2018.

[34] Z. Shao, P. Wu, Y. Gao, I. Gutman, and X. Zhang, "On the maximum ABC index of graphs without pendent vertices," Applied Mathematics and Computation, vol. 315, pp. 298-312, 2017.

[35] S. Wang, Z. Shao, J.-B. Liu, and B. Wei, "The bounds of vertex Padmakar-Ivan index on k-trees," Mathematics, vol. 7, no. 4, pp. 324-333, 2019.

[36] Z. Shao, M. Liang, and X. Xu, "Some new optimal generalized Sidon sequences," Ars Combinatoria, vol. 107, pp. 369-378, 2012.

[37] Z. Shao, J. Amjadi, S. M. Sheikholeslami, and M. Valinavaz, "On the total double roman domination," IEEE Access, vol. 7, pp. 52035-52041, 2019.

[38] J.-B. Liu, C. Wang, S. Wang, and B. Wei, “Zagreb indices and multiplicative Zagreb indices of eulerian graphs," Bulletin of the Malaysian Mathematical Sciences Society, vol. 42, no. 1, pp. 67-78, 2019.

[39] J.-B. Liu, J. Zhao, and Z. X. Zhu, "On the number of spanning trees and normalized Laplacian of linear octagonal quadrilateral networks," International Journal of Quantum Chemistry, vol. 119, p. 25971, 2019.

[40] Y. Li, F. Li, Z. Zhou, and Z. Chen, " $\mathrm{SiC}_{2}$ silagraphene and its one-dimensional derivatives: where planar tetracoordinate silicon happens," Journal of the American Chemical Society, vol. 133, no. 4, pp. 900-908, 2011.

[41] L.-J. Zhou, Y.-F. Zhang, and L.-M. Wu, " $\mathrm{SiC}_{2}$ Siligraphene and nanotubes: novel donor materials in excitonic solar cells," Nano Letters, vol. 13, no. 11, pp. 5431-5436, 2013.

[42] X. Li and I. Gutman, "Mathematical aspects of Randićtype molecular structure descriptors," Mathematical Chemistry Monographs, Kragujevac, Serbia, 2006. 